TITLE:

\title{
Fundamental properties of substorm time energetic electrons in the inner magnetosphere
}

\author{
$\operatorname{AUTHOR}(\mathrm{S})$ :
}

Ebihara, Y.; Tanaka, T.

\section{CITATION:}

Ebihara, Y....[et al]. Fundamental properties of substorm time energetic electrons in the inner magnetosphere. Journal of Geophysical Research: Space Physics 2013, 118(4): 15891603

ISSUE DATE:

2013-04

URL:

http://hdl.handle.net/2433/193966

RIGHT:

(C)2013. American Geophysical Union. 


\title{
Fundamental properties of substorm time energetic electrons in the inner magnetosphere
}

\author{
Y. Ebihara ${ }^{1}$ and T. Tanaka ${ }^{2}$ \\ Received 10 July 2012; revised 27 December 2012; accepted 4 January 2013; published 26 April 2013.
}

[1] We studied fundamental properties of spatial-temporal evolution of energetic electrons trapped in the inner magnetosphere $(L \leq 7.4)$ during an isolated substorm by using a four-dimensional drift kinetic simulation under the time-dependent electric and magnetic fields provided by a global magnetohydrodynamics (MHD) simulation. When the interplanetary magnetic field turns southward, both the potential and induction electric fields start to increase in the inner magnetosphere, resulting in a gradual injection of low-energy electrons $(\leq 51.9 \mathrm{keV})$ and deceleration of high-energy electrons $(\geq 114 \mathrm{keV})$. The deceleration of high-energy electrons results in a decrease in the phase space density of the high-energy electrons during the growth phase. After a while, an abrupt transition of phase state (a substorm onset) occurs in the magnetosphere, which triggers abrupt changes in the magnetosphere and ionosphere. The $A L$ index decreases rapidly, and magnetic field lines become dipole-like. The dipolarization does not proceed smoothly in the inner magnetosphere because of significant force imbalance between the $J \times B$ force and the grad $P$ force. As a consequence, the electric field oscillates with a period of 2-3 min, resulting in multiple injections of the low-energy electrons. The low- and high-energy electrons are accelerated under the strong influence of the drift betatron and gyro betatron, so that the acceleration process is essentially nonlinear. Our simulation results suggest that the force-induced processes play an essential role in the substorm-associated redistribution of energetic particles in the inner magnetosphere.

Citation: Ebihara, Y., and T. Tanaka (2013), Fundamental properties of substorm time energetic electrons in the inner magnetosphere, J. Geophys. Res. Space Physics, 118, 1589-1603, doi:10.1002/jgra.50115.

\section{Introduction}

[2] A substorm is known to be accompanied by large variations in the charged particles trapped in the inner magnetosphere at energy ranges from $\sim \mathrm{eV}$ to tens of $\mathrm{keV}$ [e.g., Freeman and Maguire, 1967; Arnoldy and Chan, 1969; DeForest and McIlwain, 1971; Erickson and Winckler, 1973; McIlwain, 1974; Walker et al., 1976; Sauvaud and Winckler, 1980; Birn et al., 1997], from tens of $\mathrm{keV}$ to hundreds of keV [e.g., Hones et al., 1968; Baker et al., 1982a; Lopez et al., 1990; Friedel et al., 1996; Reeves et al., 1996; Birn et al., 1997, 1998; Sergeev et al., 1998], and of $\sim \mathrm{MeV}$ [e.g., Nagai, 1988; Nagai et al., 2006]. A variety of modeling efforts has been conducted to explain the substorm time variations of energetic electrons in the inner magnetosphere. McIlwain [1974] proposed a global distribution of the electric field that could have transported electrons to the observed location at the geosynchronous orbit. By tracing the electron's trajectory backward in time, McIlwain [1974]

\footnotetext{
${ }^{1}$ Research Institute for Sustainable Humanosphere, Kyoto University, Uji, Japan.

${ }^{2}$ Kyushu University, Fukuoka, Japan.

Corresponding author: Y. Ebihara, Research Institute for Sustainable Humanosphere, Kyoto University, Uji, Japan. (ebihara@rish.kyoto-u.ac.jp)

(C)2013. American Geophysical Union. All Rights Reserved. 2169-9380/13/10.1002/jgra.50115
}

identified a longitudinally elongated boundary, which is called an injection boundary. Li et al. [1998] incorporated pulsed electric and magnetic fields propagating earthward to show an abrupt increase in energetic electrons. Fok et al. [1999, 2001] demonstrated a sudden appearance of hot plasma and relativistic electrons by stretching and dipolarizing the magnetic field according to an empirical magnetic field model. Using the Rice Convection Model, Zhang et al. [2009] showed that an earthward-propagating bubble from the magnetotail pushes magnetic flux tubes, increasing the plasma pressure near the geosynchronous orbit at midnight. Glocer et al. [2011] showed that by coupling the Radiation Belt Environment model [Fok et al., 2001, 2008; Zheng et al., 2003] with the Block-Adaptive-Tree Solar Wind Roe-Type Upwind Scheme code [Powell et al., 1999; De Zeeuw et al., 2000; Gombosi et al., 2003], the inductive electric field associated with dipolarization is responsible for the acceleration of relativistic electrons. The acceleration of charged particles is also significant in the near-Earth plasma sheet as was demonstrated by Birn et al. [1998]. They showed that electrons undergo betatron acceleration in the collapsing magnetic field earthward of the near-Earth plasma sheet. The accelerated particles in the near-Earth plasma may influence the variations near the geosynchronous orbit [Kim et al., 2000].

[3] In spite of numerous studies, very few causality arguments have been made to answer the fundamental question of what the root cause of the variations of energetic particles 
in the inner magnetosphere is. It is quite obvious that charged particles are accelerated by the electric field. Why is the electric field substantially changed during the substorm? In this paper, we focus on the variation in the electric and magnetic fields and its influence on energetic electrons in the inner magnetosphere during an isolated substorm. A recently developed state transition model of a substorm is employed, in which the generation of a pressure regime and the accompanying partial ring current are direct causes of the onset [Tanaka et al., 2010]. While the flowbraking model considers the nonmagnetohydrodynamics (MHD) effect as essential to the formation of a current wedge, the state transition model [Tanaka et al., 2010] considers the substorm as being part of the development and transition states of the convection system. We incorporate a drift kinetic simulation of energetic electrons with the global MHD simulation developed by Tanaka et al. [2010] for the purpose of demonstrating that the injection and redistribution of the energetic electrons can be understood in view of the state transition model.

\section{Simulation}

\subsection{Global MHD Simulation}

[4] The global MHD simulation of Tanaka et al. [2010] utilizes a finite-volume total variation diminishing scheme to enable adequate capture of a shock. Despite the exclusion of non-MHD processes, this simulation exhibits features that resemble those observed in the course of substorms. Characteristic features include (1) the formation of a near-Earth neutral line and earthward plasma flow in the near-Earth plasma sheet, (2) stretching and dipolarization of magnetic field lines with a short-lived depression as well as deflection of the azimuthal component of the magnetic field, and (3) a sudden decrease in the north-south component of the ground magnetic field at the auroral latitude. A detailed description of the global MHD simulation used in this study is found in Tanaka et al. [2010]. Although many arguments have been put forth in favor of nonideal MHD processes [e.g., Lui et al., 1999], we believe that the use of the global MHD simulation is one of the most reasonable means of self-consistently describing the electric and magnetic fields to solve the kinetic equation for a trapped particle population in the inner magnetosphere.

[5] The parameters used to drive the global MHD simulation are slightly different from those used by Tanaka et al. [2010]. The solar wind velocity and density are constant in time and set to $(-372.4,0,0) \mathrm{km} / \mathrm{s}$ and $10 \mathrm{~cm}^{-3}$, respectively. The interplanetary magnetic field (IMF) is changed from northward $(0,2.5,4.33) \mathrm{nT}$ to southward $(0,4.33$, $-4.33) \mathrm{nT}$ as a step function. Although the results of the global MHD simulation are also slightly different, the evolution of a substorm as well as the ensuing physical processes that occur in the magnetosphere-ionosphere system is essentially the same.

\subsection{Transport of Energetic Electrons}

\subsubsection{Drift Kinetic Equation}

[6] We solved the drift kinetic equation for the fourdimensional phase space density (PSD) $f$ as

$$
\begin{aligned}
\frac{\partial f}{\partial t} & +\frac{1}{R S_{b}} \frac{\partial}{\partial R}\left(R S_{b}\left\langle\frac{d R}{d t}\right\rangle f\right)+\frac{1}{S_{b}} \frac{\partial}{\partial \varphi}\left(S_{b}\left\langle\frac{d \varphi}{d t}\right\rangle f\right) \\
& +\frac{1}{\gamma p} \frac{\partial}{\partial K}\left(\gamma p\left\langle\frac{d K}{d t}\right\rangle f\right)+\frac{1}{x S_{b}} \frac{\partial}{\partial x}\left(x S_{b} \frac{d x}{d t} f\right)=\left(-\frac{2 f}{\tau_{b}}\right)_{\text {loss cone }},
\end{aligned}
$$

where $R=$ distance from the center of the Earth, $\varphi=$ magnetic local time (MLT), $K=$ kinetic energy, $x=$ cosine of the equatorial pitch angle, $p=$ momentum of a particle, $\gamma=$ Lorentz factor, and $\tau_{b}=$ bounce period. The bracket pair \langle\rangle denotes the bounce-averaging operator. The right-hand side of (1) implies that particles are lost on a timescale of half the bounce period in the loss cone. $S_{b}$ is a function of the equatorial pitch angle

$$
S_{b}=\int_{s_{m^{\prime}}}^{s_{m}}\left(1-\frac{B(s)}{B_{m}}\right)^{-1 / 2} d s
$$

where $S_{m}$ and $S_{m^{\prime}}=$ mirror points in the Northern and Southern Hemispheres, respectively, and $B_{m}=$ magnetic field at the mirror point. The momentum of the particle is given by

$$
p=\frac{\sqrt{K^{2}+2 m c^{2} K}}{c},
$$

where $m=$ rest mass and $c=$ speed of light. For the derivation of equation (1), refer to Appendix A.

[7] Following Roederer [1970], the bounce-averaged drift velocity and the change in the kinetic energy are given by

$$
\langle\mathbf{V}\rangle=\frac{p^{2}}{q m_{0} S_{b} B_{0}^{2}} \nabla I \times \mathbf{B}_{0}+\frac{\mathbf{E}_{0} \times \mathbf{B}_{0}}{B_{0}^{2}},
$$

where $q=$ charge and $\mathbf{E}_{0}$ and $\mathbf{B}_{0}=$ electric and magnetic fields in the equatorial plane, respectively. $I$ is a function of the equatorial pitch angle, which is given by

$$
I=\int_{s_{m}}^{s_{m}}\left(1-\frac{B(s)}{B_{m}}\right)^{1 / 2} d s .
$$

[8] The change in the kinetic energy is given by

$$
\left\langle\frac{d K}{d t}\right\rangle=\frac{M}{\gamma} \frac{\partial\langle B\rangle}{\partial t}+q\langle\mathbf{V}\rangle \bullet \mathbf{E}_{0},
$$

where $M$ is the relativistic magnetic moment given by

$$
M=\frac{p^{2}\left(1-x^{2}\right)}{2 m B} .
$$

[9] The first term on the right-hand side of equation (6) describes the gyro-betatron acceleration, and the second term describes the drift-betatron acceleration. Roederer [1970] showed that

$$
\langle B\rangle=B_{m}\left(1-\frac{I}{S_{b}}\right) .
$$

[10] The change in $x$ (cosine of the equatorial pitch angle) is given by 
$\frac{d x}{d t}=\frac{1-x^{2}}{x}\left[\frac{K+m c^{2}}{K^{2}+2 m c^{2} K}\left\langle\frac{d K}{d t}\right\rangle-\frac{1}{2 B_{0}}\left(\frac{\partial B_{0}}{\partial t}+\langle\mathbf{V}\rangle \bullet \nabla B_{0}\right)\right]$.

[11] The derivation of equation (9) is in Appendix B.

[12] The drift kinetic equation is solved by the Lax-Wendroff scheme [Lax and Wendroff, 1960] with the Superbee flux limiter [Roe, 1985]. For this particular simulation, the kinetic energy ranged from $1 \mathrm{keV}$ to $4 \mathrm{MeV}$, and the radial distance in the equatorial plane ranged from 4.0 to 7.4 Earth radii $(\mathrm{Re})$. The electric and magnetic fields are given by the global MHD simulation developed by Tanaka et al. [2010].

\subsubsection{Initial Conditions}

[13] We employed the AE8MAX [Vette, 1991] empirical model, which determines the omnidirectional flux $(\Phi)$ of electrons in the energy range from $40 \mathrm{keV}$ to $7 \mathrm{MeV}$ as a function of kinetic energy, $L$ value, and $B / B_{0}$ ratio. By using the omnidirectional flux given by the AE8MAX model $(\Phi)$, we obtained the PSD of electrons as

$$
f=\frac{\Phi}{4 \pi p^{2}} .
$$

[14] As for low-energy electrons $(<30 \mathrm{keV})$, we also used the empirical model of electrons [Hardy et al., 1985]. The Hardy et al. [1985] model determines the averaged energy and number flux of precipitating electrons as a function of $K p$, magnetic latitude, and MLT. By assuming isotropic pitch angle distribution and Maxwellian distribution, we derived the PSD numerically. The PSD at high energies (given by AE8MAX) and low energies (given by the Hardy et al. [1985] model) are connected by linear interpolation. The condition corresponding to $K p=0$ is used as the initial condition because we started the simulation with the magnetically quiet condition.

\subsubsection{Boundary Conditions}

[15] We applied the Lorentzian distribution-otherwise known as the kappa distribution [Vasyliunas, 1968]- to the PSD at the outer boundary located at 7.4 Re on the nightside. The relativistic Lorentzian distribution is given by [Xiao et al., 1998]

$$
f=\frac{n \Gamma(\kappa+1)}{\pi^{2 / 3} \theta_{\perp}^{2} \theta_{\|} \kappa^{3 / 2} \Gamma(\kappa-1 / 2)}\left(1+\frac{p_{\|}^{2}}{\kappa \theta_{\|}^{2}}+\frac{p_{\perp}^{2}}{\kappa \theta_{\perp}^{2}}\right)^{-(\kappa+1)},
$$

where $n=$ number density, $\Gamma=$ gamma function, and $\kappa(>3 / 2)$ $=$ spectral index. Quantities $\theta_{\|}$and $\theta_{\perp}$ are given by

$$
\theta_{\|}^{2}=\frac{2 \kappa-3}{\kappa} Q_{\|}
$$

and

$$
\theta_{\perp}^{2}=\frac{2 \kappa-3}{2 \kappa} Q_{\perp}
$$

where

$$
Q_{\|}=\frac{\int p_{\|}^{2} f d^{3} \mathbf{p}}{n}
$$

and

$$
Q_{\perp}=\frac{\int p_{\perp}^{2} f d^{3} \mathbf{p}}{n} .
$$

$Q_{\|}$and $Q_{\perp}$ may be related to temperature in the nonrelativistic limit, that is, $k T_{\|} \rightarrow Q_{\|} / m$ and $k T_{\perp} \rightarrow Q_{\perp} / 2 m$ [Xiao et al., 1998].

[16] In this particular simulation, the number density $(n)$ and temperature $\left(k T=k T_{\perp}=k T_{\|}\right)$are $1.7 \mathrm{~cm}^{-3}$ and $2.3 \mathrm{keV}$, respectively, which correspond to the mean values observed at the geosynchronous orbit during substorms [Birn et al., 1997]. The kappa parameter $\kappa$ is set to 5.4, which corresponds to the mean value observed during active times under the condition that $A E>100 \mathrm{nT}$ [Christon et al., 1991]. The PSD at the dayside boundary is zero.

\section{Results}

\subsection{Global MHD Simulation}

[17] Detailed analyses of a simulated substorm have already been described by Tanaka et al. [2010]. We herein focus on the evolution of the simulated substorm in the inner magnetosphere. Figure 1 shows the superposed plots of the north-south component ( $H$-component) of the ground magnetic field at 12 different MLTs at a magnetic latitude of $67^{\circ}$. Only the Hall ionospheric current is considered in the calculation. The contribution from the induction in the ground is excluded. The upper and lower envelopes of the plots may correspond to the $A U$ and $A L$ indices, respectively [Davis and Sugiura, 1966]. Epoch time zero $(T=0)$ is defined as the time when the southward IMF reaches the bow shock at the subsolar point. At $T \approx 6 \mathrm{~min}$, the $A L$ index starts to decrease and then remains almost constant until $T \approx 51 \mathrm{~min}$. At $T \approx 51 \mathrm{~min}$, it starts to decrease abruptly. Although the substorm phases should be identified by the morphological evolution of auroral structures [Rostoker et al., 1980], we define the substorm onset in terms of the abrupt decrease in the $A L$ index, that is, at $T \approx 51 \mathrm{~min}$. For convenience, we call the period between $T \approx 6$ and $51 \mathrm{~min}$ the growth phase, the period between $T \approx 51$ and $60 \mathrm{~min}$

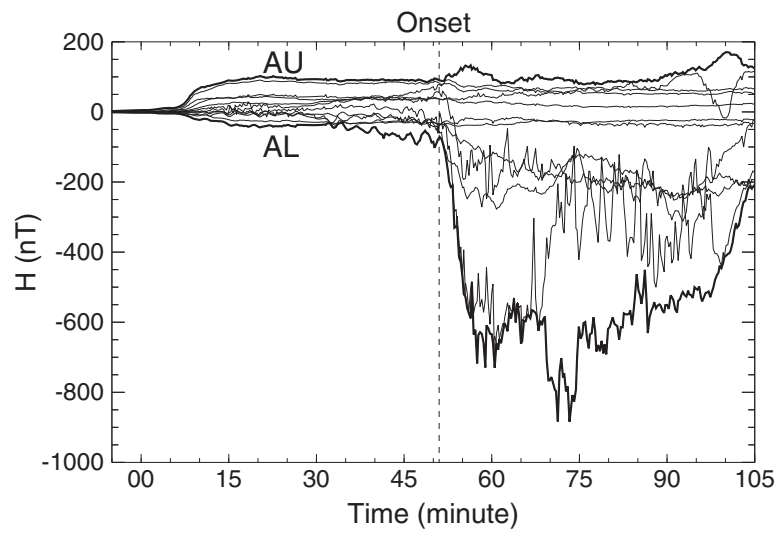

Figure 1. North-south component ( $H$-component) of the magnetic field disturbance on the ground at $0,2,4, \ldots$, and 22 MLTs at a magnetic latitude of $67^{\circ}$. The upper and lower envelopes of the superposed plots, as indicated by thick lines, correspond to the $A U$ and $A L$ indices, respectively, by definition. 


\section{EBIHARA AND TANAKA: IMPACT OF SUBSTORM ON ENERGETIC ELECTRON}

the expansion phase, and the period from $T \approx 60$ min onward the recovery phase.

[18] Figure 2 shows the temporal variation in the selected magnetic field lines. After the arrival of the southward IMF at the bow shock $(T=0)$, the magnetic field lines become highly stretched. During the growth phase, earthward magnetic tension balances with the tailward pressure gradient force throughout the plasma sheet. Just after the onset at $T \approx 51 \mathrm{~min}$, the force balance in the plasma sheet changes a configuration of dynamics. Within $9 \mathrm{Re}$, a new static force balance tends to be realized between two regions: the increased-pressure region and the dipolarizing magnetic field region. Beyond $10 \mathrm{Re}$, magnetic tension alternately balances with acceleration through a decrease in the pressure gradient. This process corresponds to the magnetic-tension-driven bulk flow, which cancels the stagnation of plasma sheet convection that remained constant during the growth phase. As a result, a high-pressure region is formed near geosynchronous altitude as indicated by the red color. Readers may refer to Tanaka et al. [2010] for a detailed explanation.

[19] Figure 3 shows the plasma pressure $(P)$ and $Y$ component of the electric field $\left(E_{y}\right)$ as a function of the radial distance and time at midnight in the equatorial plane. The plasma pressure appears to increase significantly in the region between $\sim 6$ and $\sim 8 \mathrm{Re}$. After the onset, the highpressure region evolves to become tailward and earthward. A black curve represents a streamline of the ionospheric

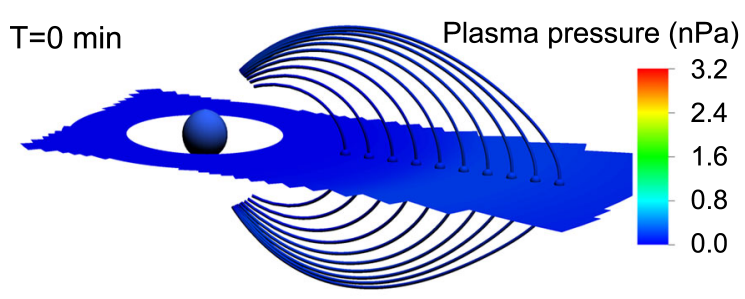

$\mathrm{T}=36 \min$

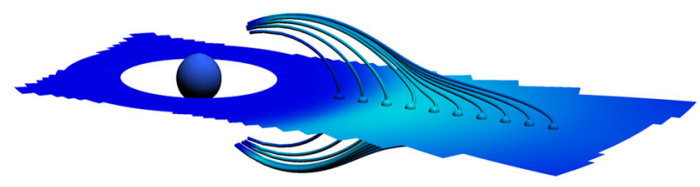

$\mathrm{T}=52 \min$

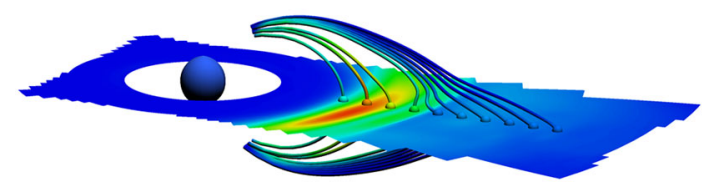

$\mathrm{T}=58 \mathrm{~min}$

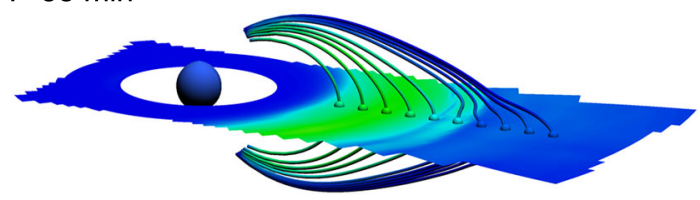

Figure 2. Magnetic field lines intersecting the equatorial plane at radial distances of $6,7, \ldots$, and $15 \mathrm{Re}$ at midnight. From top to bottom, magnetic field lines are shown at $T=0$, 36,52 , and $58 \mathrm{~min}$, respectively. The color code indicates the plasma pressure. The sphere indicates the Earth.

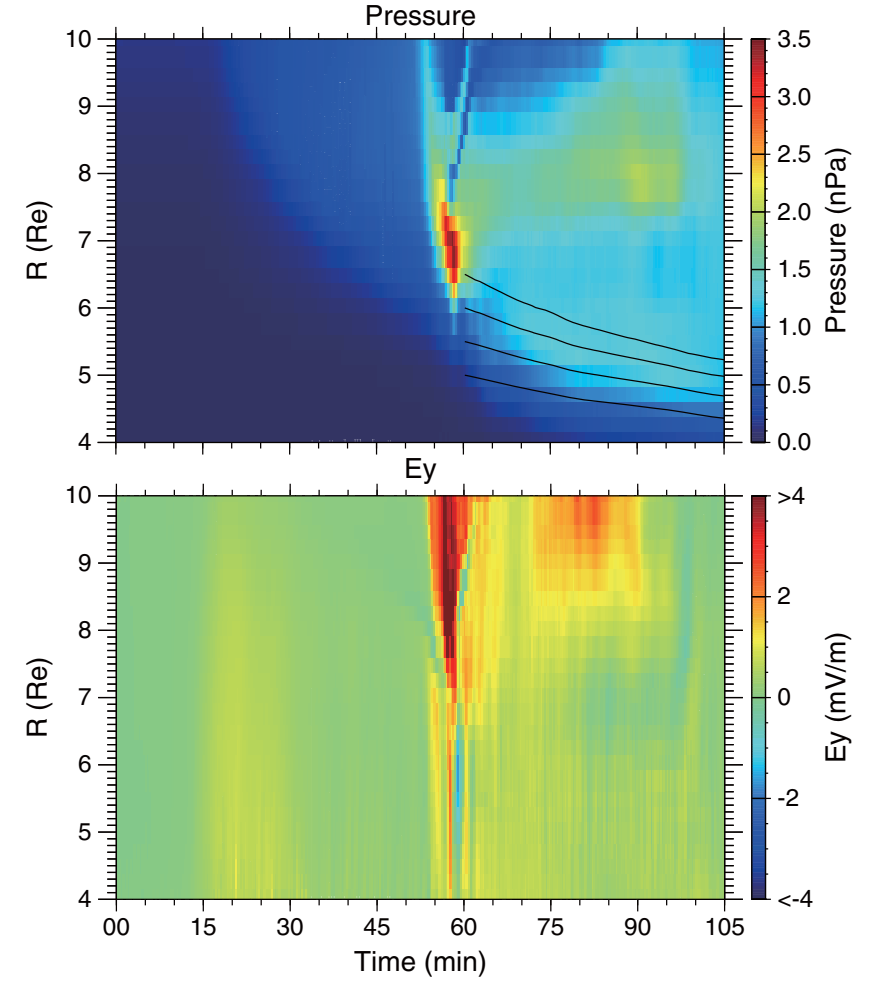

Figure 3. (top) Plasma pressure, $P$, and (bottom) duskdawn component of electric fields, $E_{\mathrm{y}}$, at midnight in the equatorial plane.

plasma mapped to the equatorial plane, indicating that the earthward evolution of the high plasma pressure during the recovery phase is likely caused by the potential electric field originating from the ionosphere. $E_{y}$ shows an increase in the entire region (from 4 to $10 \mathrm{Re}$ ) about $15 \mathrm{~min}$ after the southward turning of IMF. Near the onset, $E_{y}$ shows a significant increase, followed by an oscillation at greater than $\sim 7 \mathrm{Re}$. The oscillation also appears almost coherently at less than $\sim 7$ Re with an amplitude of $\sim 1$ or $2 \mathrm{mV} / \mathrm{m}$.

[20] Figure 4 shows a close-up view of selected parameters at $7.6 \mathrm{Re}$ at midnight near the onset, including $(P)$, the north-south component of the magnetic field $\left(B_{z}\right)$, the $X$-component of the plasma velocity $\left(V_{x}\right)$, the acceleration of the plasma $\left(A_{x}\right)$, and the force density $\left(F_{x}\right)$. The force density $\mathbf{F}$ is given by

$$
\begin{aligned}
\mathbf{F} & =-\nabla P+\mathbf{J} \times \mathbf{B} \\
& =-\nabla P+\frac{(\mathbf{B} \bullet \nabla) \mathbf{B}}{\mu_{0}}-\nabla\left(\frac{B^{2}}{2 \mu_{0}}\right),
\end{aligned}
$$

where $\mu_{0}=$ magnetic constant and $\mathbf{J}=$ current density. The acceleration of the plasma is given by $\mathbf{A}=\mathbf{F} / \rho$, where $\rho$ is the mass density of plasma. The three terms on the righthand side of equation (16) are called the plasma pressure force, the tension force, and the magnetic pressure force, respectively. They are indicated by the red, blue, and green colors in Figure 4f. In the MHD simulation, the electric field $\mathbf{E}$ is given by $\mathbf{E}=-\mathbf{V} \times \mathbf{B}$. The plasma velocity $\mathbf{V}$ can be primarily changed by two factors [Tanaka, 1995]. One is the potential electric field, which is transferred from the ionosphere. The plasma velocity that corresponds to the electric 


\section{EBIHARA AND TANAKA: IMPACT OF SUBSTORM ON ENERGETIC ELECTRON}

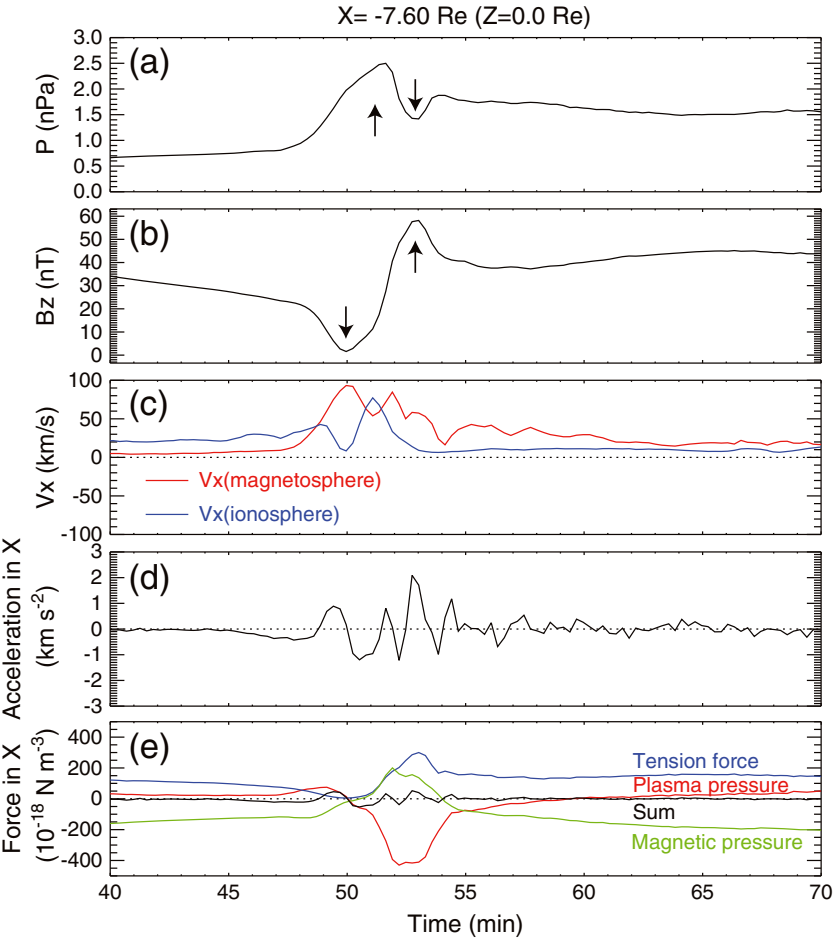

Figure 4. (a) Plasma pressure, $P$, (b) north-south component of the magnetic field, $B_{z}$, (c) $X$-component of the plasma velocity, $V_{x}$, (d) $X$-component of the acceleration, $A_{x}$, and (e) $X$-component of the force density at $7.6 \mathrm{Re}$ at midnight in the equatorial plane. In Figure $4 \mathrm{c}, V_{x}$ in the magnetosphere is indicated by the red line, and $V_{x}$ mapped from the ionosphere is indicated by the blue line.

field mapped from the ionosphere is plotted as a blue line in the third panel of Figure 4. The mapping is performed without taking into account the Alfvén transit time between the ionosphere and the magnetosphere. The other factor is the force imbalance in the magnetosphere. Near the onset, the three forces are significantly changed (as shown in Figure 4e), resulting in the force imbalance that accelerates the plasma (as shown in Figure 4d). The positive (negative) value of $A_{x}$ corresponds to the increase (decrease) in $V_{x}$. It is interesting to note that $A_{x}$ and $V_{x}$ show oscillation with a period of $\sim 2 \mathrm{~min}$, whereas each force term (plasma pressure force, tension force, and magnetic pressure force) does not show such quasiperiodic oscillation. The oscillations in $A_{x}$ and $V_{x}$ are not simply due to the temporal variation of a single force term. The oscillations are caused by the motion of perturbed distributions of $P$ and $\mathbf{B}$ in the state transition phase in the inner magnetosphere. The cause of the oscillation may be described as follows.

[21] Figures 5 and 6 show radial profiles of magnetic field, plasma pressure, $X$-component of the acceleration of the plasma, and $X$-component of the force density at midnight in the equatorial plane. The red, blue, and green lines indicate the plasma pressure force, $F_{P}$, the tension force, $F_{T}$, and the magnetic pressure force, $F_{B}$, respectively. The dashed black line indicates the sum of the three forces, and the solid black one indicates that calculated by the equation $\mathbf{F}=-\nabla P+\mathbf{J} \times \mathbf{B}$. The dashed black line must be identical with the solid one, but they are slightly different from each other because of numerical error.

[22] We focus on the $X$-component of the acceleration of the plasma, $A_{x}$, at $7.6 \operatorname{Re}(X=-7.6 \mathrm{Re})$ in the equatorial plane.

(a) At $T=48.03 \mathrm{~min}, A_{x}$ is negative because of a decrease in $F_{B}$ (large negative magnetic pressure force).

(b) At $T=49.14 \mathrm{~min}, A_{x}$ is positive because of an increase in $F_{P}$.

(c) At $T=50.52 \mathrm{~min}, A_{x}$ is negative because of a decrease in $F_{P}$.

(d) At $T=51.63 \mathrm{~min}, A_{x}$ is positive because of an increase in $F_{B}$ and an increase in $F_{T}$.

(e) At $T=52.19 \mathrm{~min}, A_{x}$ is negative because of a decrease in $F_{P}$.

(f) At $T=52.74 \mathrm{~min}, A_{x}$ is positive because of an increase in $F_{T}$.

(g) At $T=53.85 \mathrm{~min}, A_{x}$ is negative because of a decrease in $F_{T}$ and a decrease in $F_{B}$.

(h) At $T=54.41 \mathrm{~min}, A_{x}$ is positive because of an increase in $F_{P}$.

[23] The oscillation may be divided into two in terms of the structures of $P$ and $B$ : the early period from (a) to (d) and the latter period from (e) to (h). Figure 7 shows a schematic diagram of the relative location to the structure of $B, P$, and $F_{T}$. During the early period from (a) to (d), the plasma pressure is significantly enhanced by the following two-step process. First, the magnetic tension released from the nearEarth plasma sheet generates the convergence of the thermal energy flux at 8-15 Re. Secondly, the convergence of the thermal energy flux is transported to the inner magnetosphere at 6-8 Re [Tanaka et al., 2010]. $F_{P}$ looks outward from the peak of the plasma pressure. The plasma pressure causes the diamagnetic current that reduces $B$ near the peak of the plasma pressure. The reduction of $B$ results in $F_{B}$ looking toward the minimum of $B . F_{T}$ also contributes to the earthward force in the outer part of the region of interest. Thus, it can be said that the high pressure is sustained (or sandwiched) by the magnetic pressure and the tension force. The peak of $P$ moves earthward. An observer who is in a fixed frame of reference experiences (a) the tailward force by $F_{B}$ first, followed by (b) earthward force by $F_{P}$, (c) tailward force by $F_{P}$, and (d) earthward force by $F_{B}$ and $F_{T}$. In short, the observer experiences two cycles of oscillation when traversing the prime peak of $P$.

[24] During the latter period from (e) to (h), the second peak of $P$ appears tailward of the prime peak, which is caused by the flow breaking [Tanaka et al., 2010]. Two humps of $P$ result in the reversed normal vector of the field line curvature in the outer part of the region of interest, that is, $F_{T}$ looking outward, as shown in Figure 8. In addition to the tailward force of $F_{B}$, the plasma moves tailward, and consequently, the second pressure peak retreats tailward. The observer experiences (e) the tailward force by $F_{P}$ first, followed by (f) the earthward force by $F_{T}$ due to the stretched field line, $(\mathrm{g})$ the tailward force by $F_{P}$ with a reduction of $F_{T}$ and $F_{B}$, and (h) the earthward force by $F_{T}$ with a reduction of $F_{P}$. In short, the observer experiences two cycles of oscillation when traversing the negative gradient of the prime peak of $P$ from outside to inside after the establishment of the second peak of $P$.

[25] Figure 9 is almost the same as Figure 4 except at 5.4 Re. $V_{x}$ shows a strong oscillation with a period of $\sim 2-3 \mathrm{~min}$ in the magnetosphere. Until $53 \mathrm{~min}, V_{x}$ is almost positive because of the influence of the potential electric field 
EBIHARA AND TANAKA: IMPACT OF SUBSTORM ON ENERGETIC ELECTRON
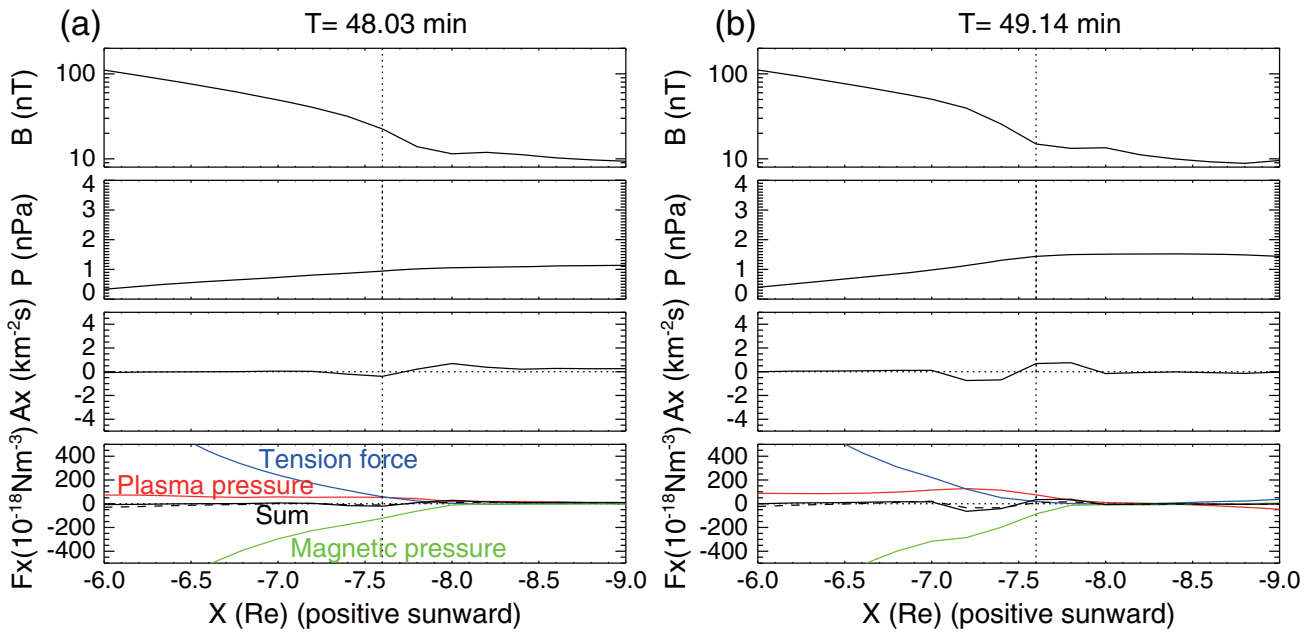

(c)

$\mathrm{T}=50.52 \mathrm{~min}$

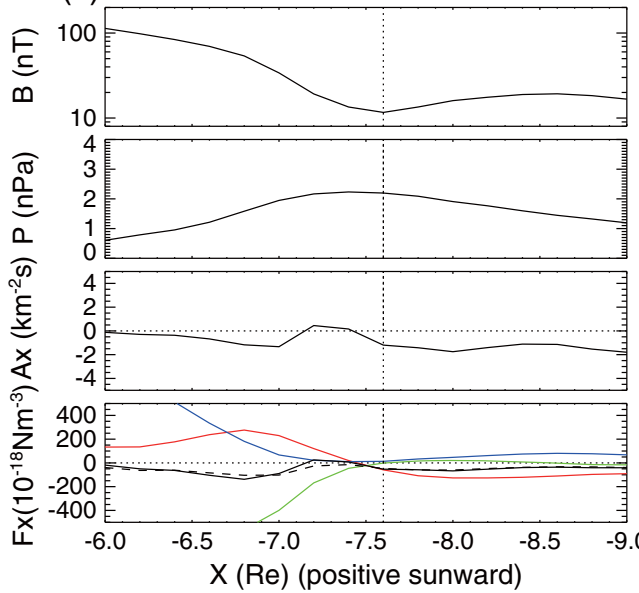

(d)

$\mathrm{T}=51.63 \mathrm{~min}$

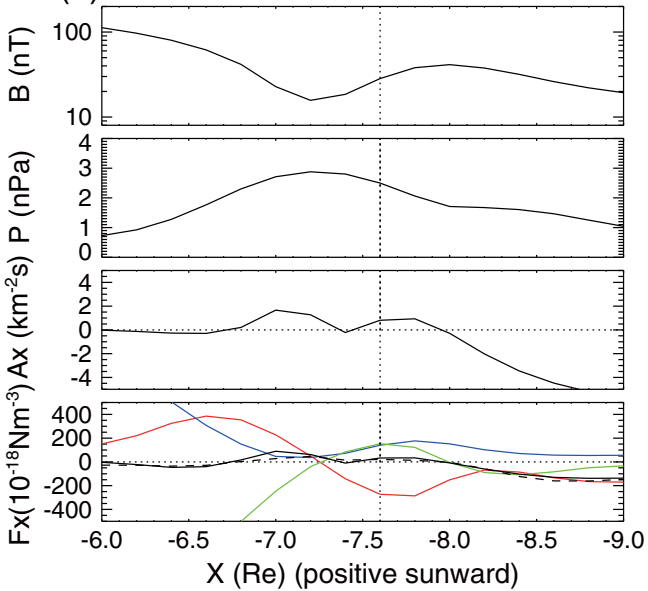

Figure 5. Magnetic field, $B$, plasma pressure, $P, X$-component of the acceleration of the plasma, $A_{x}$, and $X$-component of the force density at midnight in the equatorial plane at (a) 48.03, (b) 49.14, (c) 50.52, and (d) $51.63 \mathrm{~min}$. In the bottom panel, the red, blue, and green lines indicate the plasma pressure force, the tension force, and the magnetic pressure force, respectively. The black line indicates the sum of the three force terms.

transferred from the ionosphere. Both $P$ and $B_{z}$ increase almost simultaneously around $T \approx 51,53$, and $56 \mathrm{~min}$. This means that they are positively correlated with each other. This tendency is opposite to that seen at $7.6 \mathrm{Re}$ as shown in Figure 4. Namely, $P$ and $B_{z}$ are negatively correlated with each other at $7.6 \mathrm{Re}$, which may be regarded as the diamagnetic or slow-mode nature, caused by the sudden increase in the plasma pressure. The anticorrelation (as seen at 7.6 Re) and correlation (at $5.4 \mathrm{Re}$ ) between $P$ and $B$ are discussed by Fujita and Tanaka ("Possible generation mechanisms of Pi2 pulsations based on a global MHD simulation," Earth, Planets and Space, in press) as a possible generation mechanism of Pi2 pulsations.

[26] Figure 10 shows the correlation coefficient between $P$ and $B$ in the equatorial plane during the interval between 50 and $60 \mathrm{~min}$. It is clear that the correlation coefficient is almost 1 at $<6$ Re between $\approx 20$ and $\approx 04$ MLT. It is speculated that the variation in $V_{x}$ (or $\left.E_{y}\right)$ in the region where the correlation coefficient is almost 1 is associated with the fast magnetosonic wave because of the following two reasons. First, $P$ and $B$ are positively correlated with each other.
Secondly, the convergence of the thermal energy flux (which results in an increase in the plasma pressure) is very small at $<6 \operatorname{Re}$ (c.f., Figure 18 of Tanaka et al. [2010]). This probably means that there is not enough free energy in this region to generate the oscillation of $V_{x}$ (or $E_{y}$ ).

[27] Figure 11 shows the sequence of the plasma pressure $(P)$, the north-south component of the magnetic field $\left(B_{z}\right)$, and the dusk-dawn component of the electric field $\left(E_{y}\right)$ at 6.4 Re at midnight (left) and noon (right) in the equatorial plane. At midnight, $B_{z}$ shows a gradual decrease during the period between $T \approx 6$ and $\approx 48 \mathrm{~min}$, followed by a negative excursion of $B_{z}$ and a positive excursion of $P$. This anticorrelation between $B_{\mathrm{z}}$ and $P$ is consistent with the in situ observations of the Combined Release and Radiation Effects Satellite (CRRES) [Sergeev et al., 1998]. In the simulation, the decrease in $B_{z}$ may be explained by the increase in the diamagnetic current $\left(\mathbf{J}=\mathbf{B} \times \nabla P / B^{2}\right)$ [Tanaka et al., 2010]. The diamagnetic current surrounds the high-pressure region in a clockwise manner when one looks down the equatorial plane from the north, and it causes a decrease in $B_{z}$ in the high-pressure region. The localized enhancement of the 
EBIHARA AND TANAKA: IMPACT OF SUBSTORM ON ENERGETIC ELECTRON
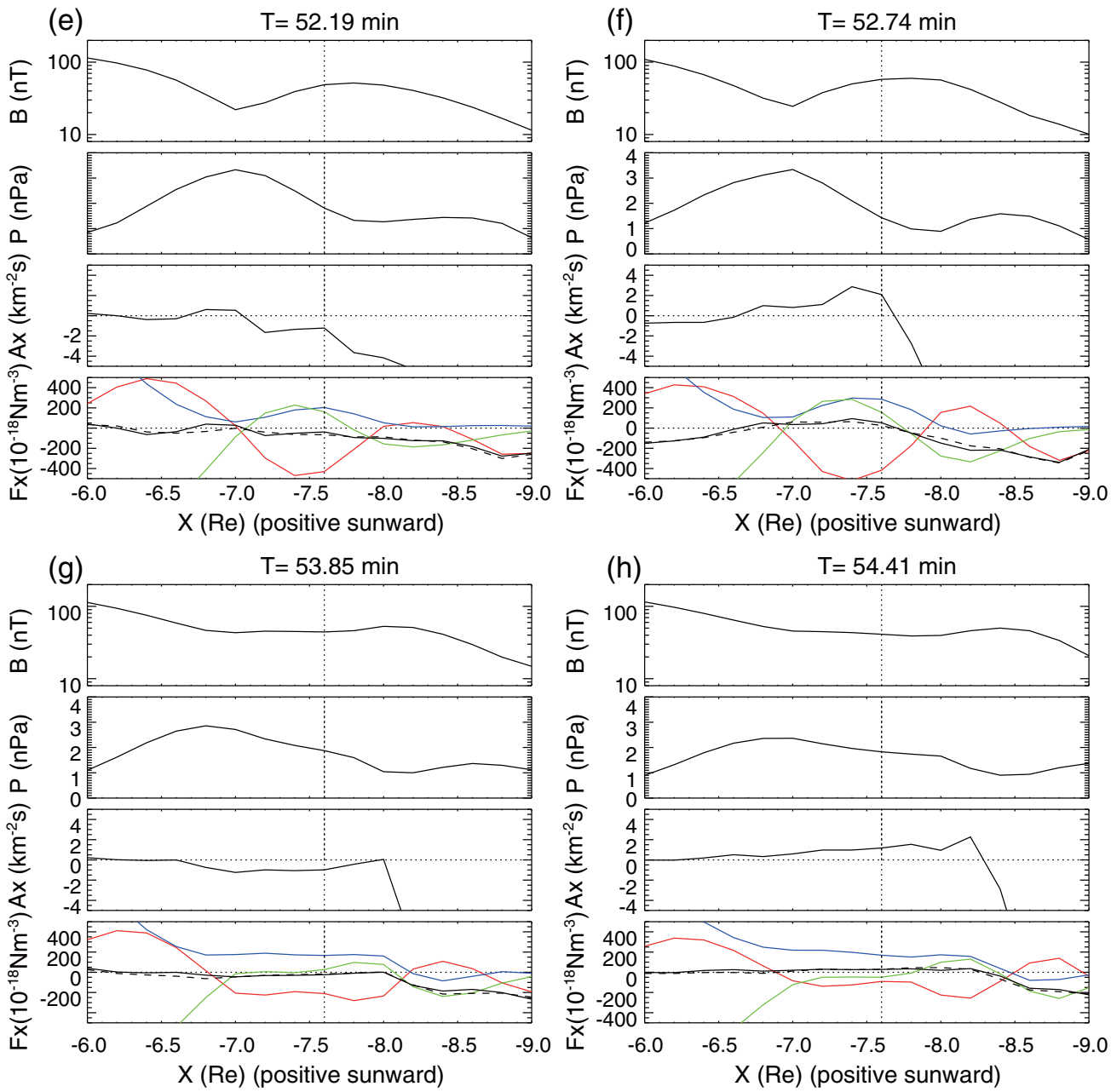

Figure 6. Same as Figure 5 except at $T=52.19,52.72,53.85$, and $54.41 \mathrm{~min}$.

pressure results in the azimuthal gradient of the diamagnetic current that is connected to the region 2 field-aligned current. This is known as the Vasyliunas-Wolf configuration [Vasyliunas, 1970; Wolf, 1970].

[28] Several minutes after the southward turning of IMF, both the magnetospheric and ionospheric electric fields start to increase almost simultaneously at midnight. The magnetospheric electric field is slightly delayed to the ionospheric electric field because of the Alfvén transit time between the ionosphere and the magnetosphere. The ionospheric electric field remains nearly unchanged during the growth phase, whereas the magnetospheric electric field decreases. The suppression of $E_{y}$ in the magnetosphere between $T \approx 15$ and $\approx 48 \mathrm{~min}$ comes from the growth of the induction electric field directing eastward. Several pulses of a largeamplitude electric field are evident in the magnetosphere near the onset between $T \approx 48$ and $55 \mathrm{~min}$. The period of the oscillation is approximately $2-3 \mathrm{~min}$, and the amplitude reaches at least $1.5 \mathrm{mV} / \mathrm{m}$. The characteristic feature of the large-amplitude fluctuations is that they resemble those previously observed by GEOS 2 [Roux et al., 1991] and CRRES [Maynard et al., 1996; Sergeev et al., 1998] in the inner magnetosphere.

[29] At noon (right panel of Figure 11), the magnetospheric electric field starts to increase immediately after the southward turning and remains almost unchanged since then. $E_{y}$ in the magnetosphere is larger than mapped from the ionosphere, implying the existence of the induction electric field directing the $Y$-direction, that is, eastward.

\subsection{Drift Kinetic Simulation}

\subsubsection{General Overview}

[30] Figure 12 summarizes the snapshots of the PSD of $10.7,35.0,252 \mathrm{keV}$, and $1.2 \mathrm{MeV}$ electrons with an equatorial pitch angle of $58.2^{\circ}$ at $T=30.00$ (growth phase), 47.00 (just before the onset), 53.25 (expansion phase), and $90.00 \mathrm{~min}$ (recovery phase). At $T=30.00 \mathrm{~min}$, the PSD of low-energy (10.7 and $35.0 \mathrm{keV}$ ) electrons is highly enhanced near the outer boundary on the nightside. The radial distance of the inner edge of the high-PSD region increases with MLT. The distribution of the high-PSD region is almost kept constant just before the onset $(T=30.00 \mathrm{~min})$. The enhancement of the PSD of low-energy electrons results from freshly transported ones coming from the nightside boundary. From the onset and onward, the PSD of 10.7 and $35.7 \mathrm{keV}$ electrons further increases. As for high-energy $(252 \mathrm{keV}$ and 1.2 MeV) electrons, the PSD is almost unchanged. One of the significant features is that the PSD of the high-energy 
EBIHARA AND TANAKA: IMPACT OF SUBSTORM ON ENERGETIC ELECTRON

Period between (a) and (d)

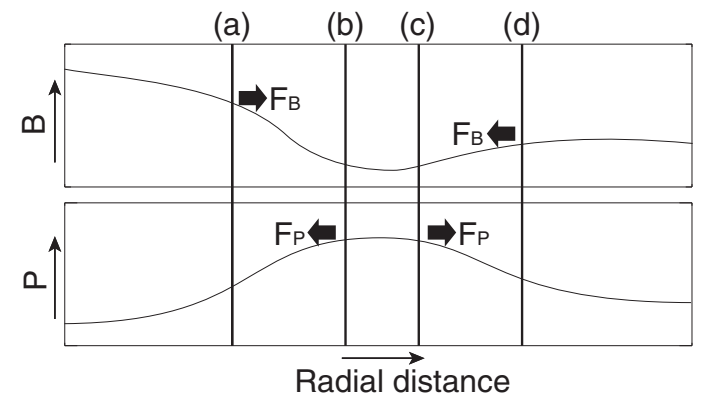

Period between (e) and $(h)$

(h) (g) (f) (e)

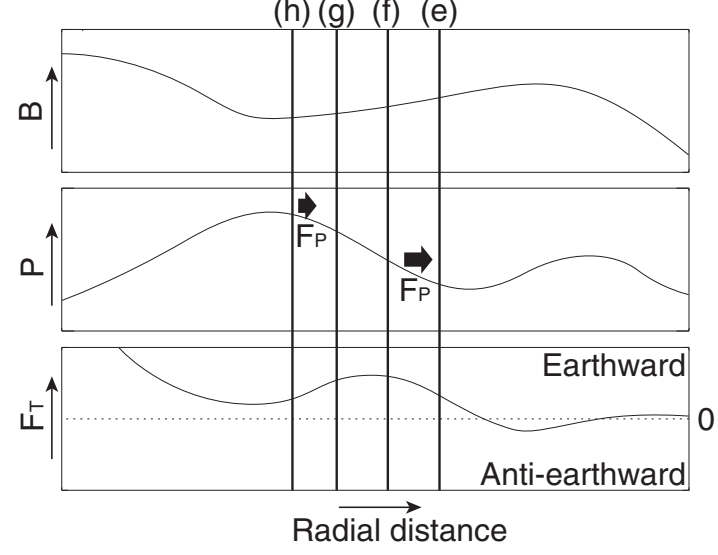

Figure 7. Schematics of the magnetic field, $B$, the plasma pressure, $P$, and the $X$-component of the tension force, $F_{T}$, as a function of radial distance from the center of the Earth.

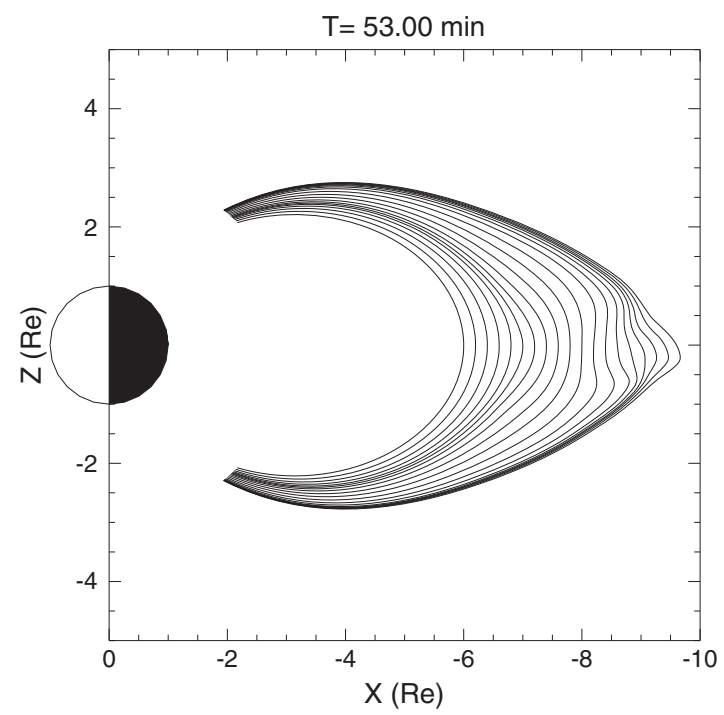

Figure 8. Magnetic field lines in the meridional plane at midnight at $53.00 \mathrm{~min}$.

electrons is significantly low near the outer boundary on the nightside.

[31] Figure 13 shows the PSD of electrons as a function of $L$-value and time at midnight. At $10.7 \mathrm{keV}$, the PSD shows a two-step increase in space and time. The first increase occurs several minutes after the southward turning of IMF,

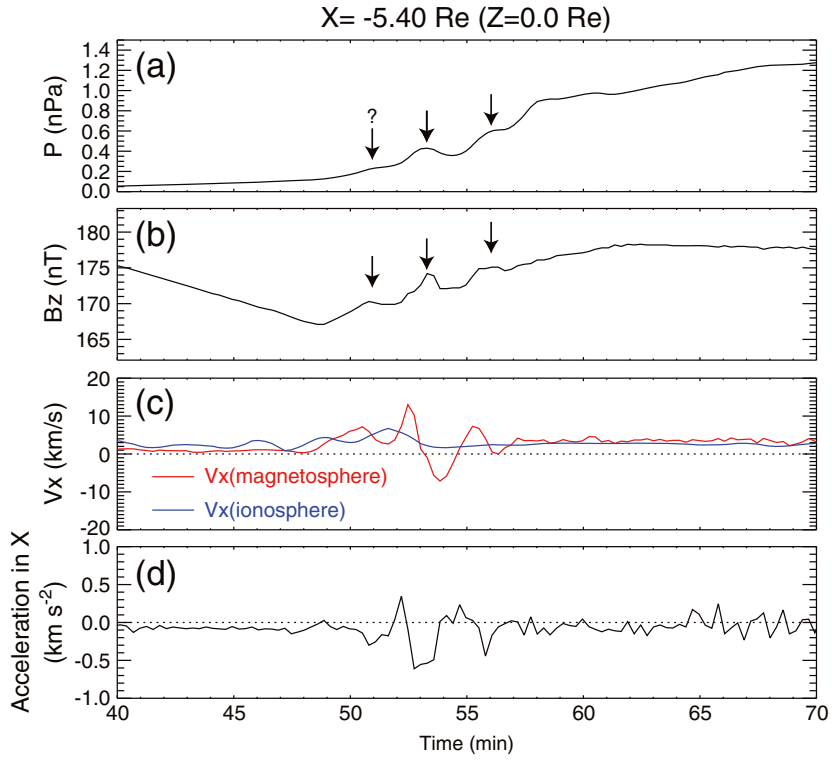

Figure 9. Almost the same as Figure 4 except at 5.4 Re.

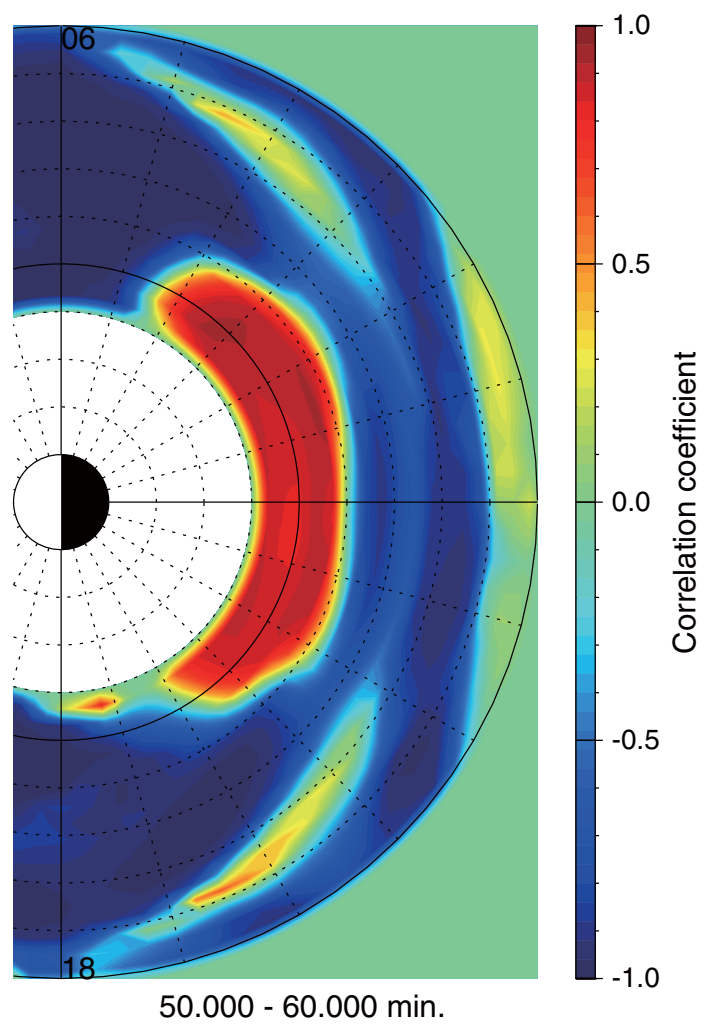

Figure 10. Correlation between the plasma pressure, $P$, and the magnetic field, $|\mathbf{B}|$, in the equatorial plane.

corresponding to the enhancement of the magnetospheric electric field on the nightside. The second one occurs near the onset. The second increase in the PSD propagates earthward from the expansion phase and onward. At $35.0 \mathrm{keV}$, the second increase is restricted at $L \geq 5.7$. At $252 \mathrm{keV}$ and 1.2 MeV, the PSD near the outer boundary significantly decreases. The decrease is most prominent near the onset. 
EBIHARA AND TANAKA: IMPACT OF SUBSTORM ON ENERGETIC ELECTRON
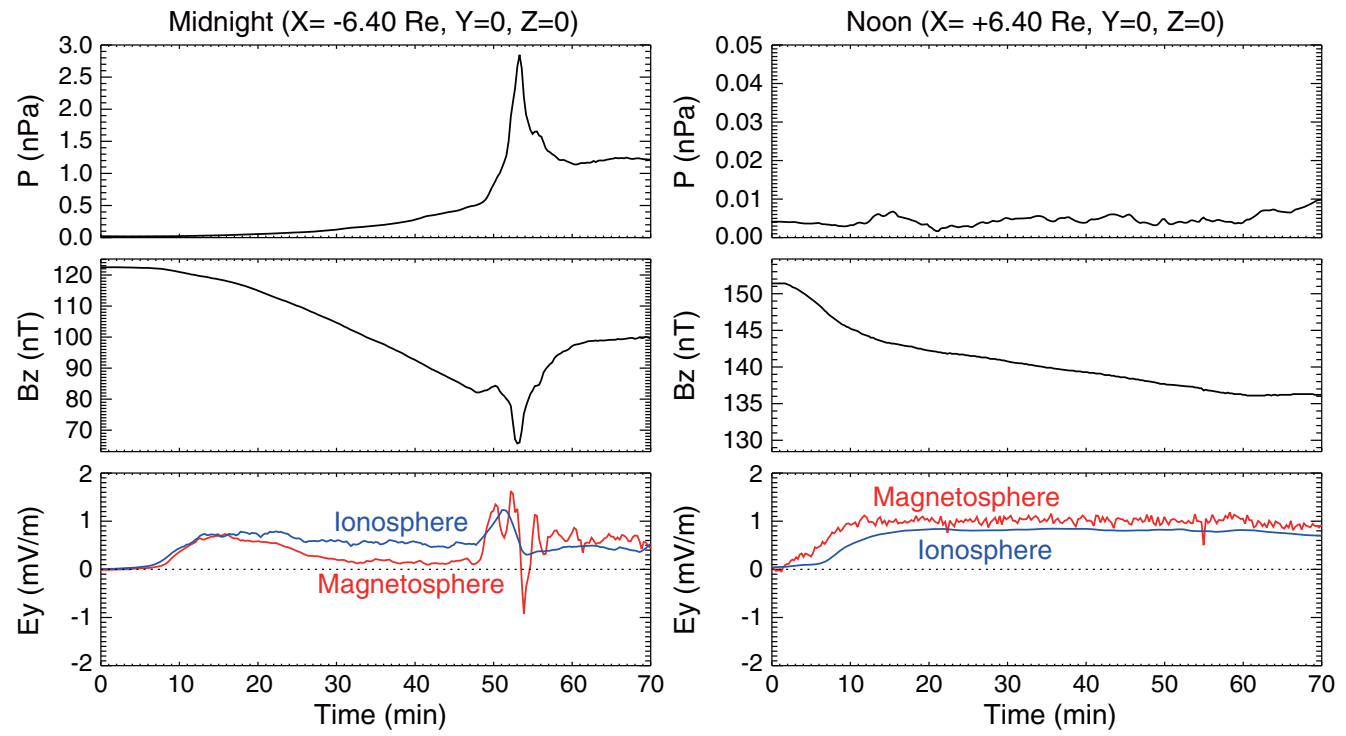

Figure 11. (top) The plasma pressure, $P$, (middle) the $Z$-component of the magnetic field, $B_{z}$, and (bottom) the $Y$-component of the electric field, $E_{y}$, at (left) midnight and (right) noon at $6.4 \mathrm{Re}$ in the equatorial plane. The magnetospheric electric field is indicated by the red color, and the ionospheric electric field mapped to the equatorial plane is indicated by the blue color.

[32] Figure 14 shows the temporal variation of the PSD of electrons at energies from $10.7 \mathrm{keV}$ to $1.2 \mathrm{MeV}$ at $L=6.34$ at midnight in the equatorial plane. The two-step increase in the PSD is prominent at energies from 10.7 to $51.9 \mathrm{keV}$. The first increase $(T \approx 22-30 \mathrm{~min})$ is monotonic, whereas the second increase $(T \approx 50-53 \mathrm{~min})$ consists of a subset of multiple increases. That is, there are at least two peaks of the PSD at $T \approx 53.5$ and $\approx 56.0 \mathrm{~min}$. As for energies from $114.4 \mathrm{keV}$ to $1.2 \mathrm{MeV}$, the PSD shows a two-step decrease. The first decrease started just after the southward turning. The second decrease occurs during the expansion phase, followed by a negative excursion which lasts for several minutes.

\subsubsection{Multiple Increases of Low-Energy Electrons}

[33] Two peaks of the PSD of low-energy electrons (from 10.7 to $51.9 \mathrm{keV}$ ) appear at $T \approx 53.5$ and $\approx 56.0 \mathrm{~min}$ as shown in Figure 14. To understand the two peaks, we tracked back in time the bounce-averaged trajectory of the $35 \mathrm{keV}$ electron that is located at $L=6.34$ at midnight in the equatorial plane at 50.0 and $53.5 \mathrm{~min}$. Hereinafter, we call this point (at $L=6.34$ at midnight in the equatorial plane) $S$. When the electron reached the outer boundary of 7.4 Re, or when the elapsed time exceeded $60 \mathrm{~min}$, the calculation was terminated. Hereinafter, $T_{1}$ and $T_{2}$ denote the start time and end time, respectively. Figure 15 shows the results. When the electron starts at point $S$ at $50.0 \mathrm{~min}$, it does not reach the outer boundary of $7.4 \mathrm{Re}$ within $60 \mathrm{~min}$, which means that the electron located at point $S$ at $50.0 \mathrm{~min}$ is a preexisting one. When the electron starts at $53.5 \mathrm{~min}$, it reaches the outer boundary, which means that the electron located at point $S$ at $53.5 \mathrm{~min}$ comes from the outer boundary, that is, a fresh one.

[34] Figure 16 shows the time history of the radial distance, kinetic energy, $K$, and energy gain rate $(\mathrm{d} K / \mathrm{d} t)$ for the $35 \mathrm{keV}$ electron that arrives at point $S$ at $53.5 \mathrm{~min}$. The kinetic energy decreases with increasing the radial distance as time proceeds backward and reaches $19 \mathrm{keV}$ at the outer boundary. According to Liouville's theorem, the PSD at $19 \mathrm{keV}$ at the boundary can be mapped to the PSD at $35 \mathrm{keV}$ at point $S$. The last panel of Figure 16 indicates that most changes of the kinetic energy come from the drift betatron except near the onset. Near the onset, the gyro betatron becomes significant because of a substantial reduction of the magnetic field, and contributions from the gyro betatron and the drift betatron are almost comparable.

[35] Figure 17 shows the kinetic energy at the end time, $T_{2}$, as a function of the start time, $T_{1}$. The kinetic energy shows slight minima around $T_{1} \approx 53.5$ and $\approx 56.0 \mathrm{~min}$ as indicated by arrows. The PSD of the relativistic Lorentzian distribution, which is imposed to the boundary, increases with decreasing kinetic energy. Lower energy at the boundary means that a larger PSD is mapped to point $S$. The bottom panel of Figure 17 shows the PSD at point $S$ calculated by the drift kinetic simulation. There are at least two maxima around $T_{1} \approx 53.5$ and $\approx 56.0 \mathrm{~min}$ as indicated by arrows, which may correspond to two minima of the kinetic energy at the boundary as shown in the top panel of Figure 17. Thus, the multiple increases in the PSD most likely result from the efficient acceleration under the contributions from the drift betatron as well as the gyro betatron. It should be noted that the kinetic energy and the PSD are not clearly anticorrelated because of the use of a finite number of numerical grids.

\subsubsection{Two-Step Decrease in High-Energy Electrons}

[36] The PSD of high-energy electrons (from $114.4 \mathrm{keV}$ to 1.2 MeV) starts to show a gradual decrease just after the southward turning, followed by a negative excursion near the onset. To understand the evolution of the PSD of the high-energy electrons, we focused on the $252 \mathrm{keV}$ electron at point $S$. The bounce-averaged trajectory of the electron was traced backward in time from point $S$ until it completed the circular motion, or it reached the outer boundary of 7.4 Re. Figure 18 shows the drift trajectories of the 
EBIHARA AND TANAKA: IMPACT OF SUBSTORM ON ENERGETIC ELECTRON
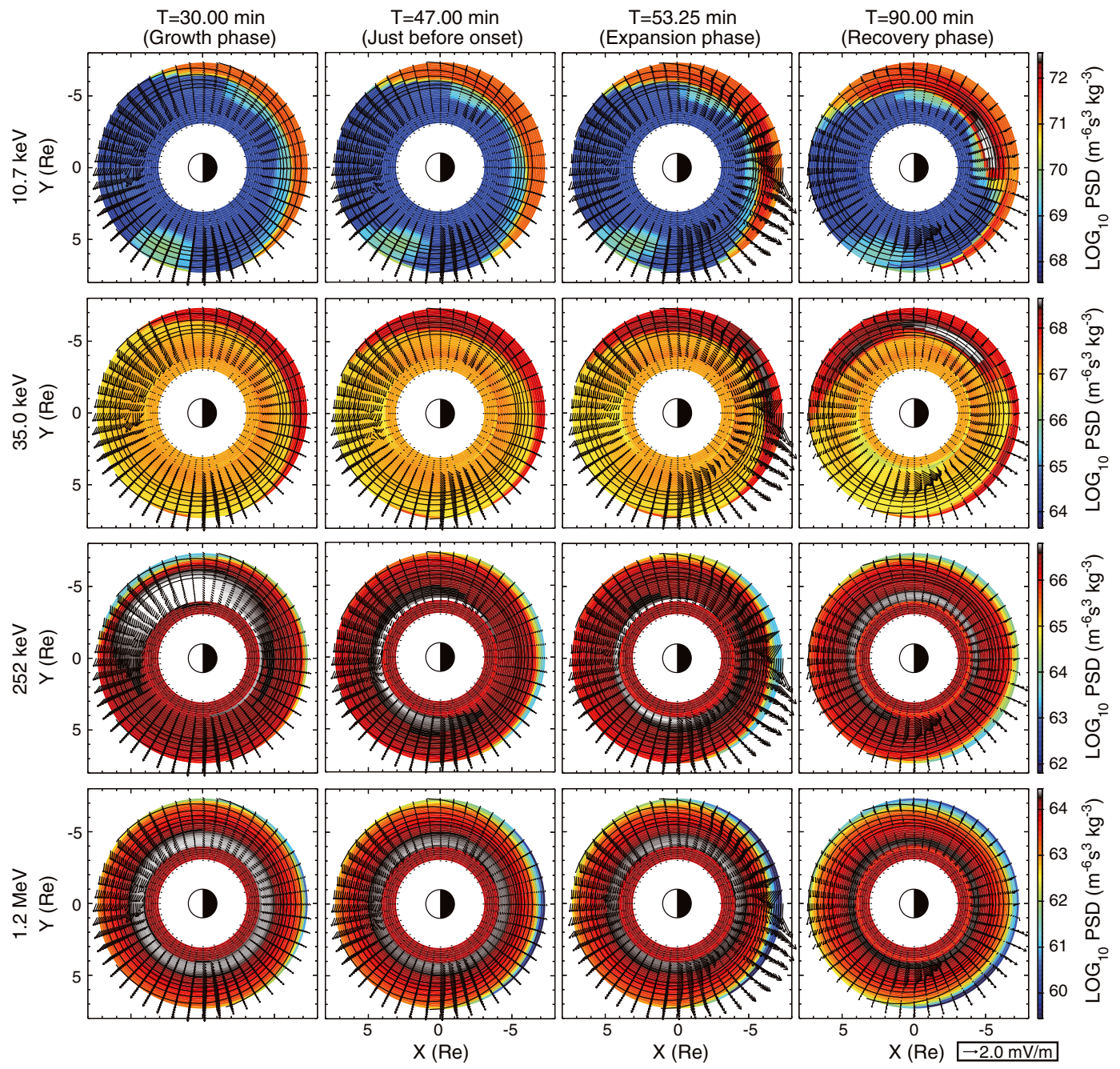

Figure 12. Snapshots of phase space density of 10.7, 35.0, 252, and $1.2 \mathrm{MeV}$ electrons in the equatorial plane. The equatorial pitch angle is $58.2^{\circ}$. Time is indicated at the top of each panel. An arrow indicates the electric field.

$252 \mathrm{keV}$ electron arriving at point $S$ at 50.0 and $53.5 \mathrm{~min}$. When an electron starts at $50.0 \mathrm{~min}$, it encircles the Earth under the strong influence of the gradient $\mathrm{B}$ and curvature drifts. In the course of the circular motion, the kinetic energy is gradually decreased, and the kinetic energy is not conserved when it completes the circular motion. This means that the eastward component of the induction electric field is present as evidenced by Figure 11. The gradual decrease of the kinetic energy results in a gradual decrease of the PSD of high-energy electrons seen at the fixed point at the fixed energy as shown in Figure 14. When the electron starts at $53.5 \mathrm{~min}$, it encounters the dayside boundary of $7.4 \mathrm{Re}$. If the PSD at the outer boundary is lower than that of preexisting electrons, the PSD will show a temporal decrease seen at the constant energy, resulting in a large decrease in the PSD during the expansion phase.

[37] Figure 19 shows a time history of the radial distance, kinetic energy, and the energy gain rate of the electron that arrives at point $S$ at $50.0 \mathrm{~min}$. As time proceeds, the radial distance is changed by the deformation of the magnetic and electric fields. When the electron completes the circular motion, it returns to near point $S$. However, the kinetic energy is decreased from $285 \mathrm{keV}$ at $T_{2}$ to $252 \mathrm{keV}$ at $T_{1}$ by the drift betatron as shown in the bottom panel of Figure 19. The gradual decrease results in the decrease in the PSD seen at the constant energy during the growth phase. The gyro betatron is not significant for this particular period.

[38] Figure 20 is the same as Figure 19 except for the electron arriving at $53.5 \mathrm{~min}$. The electron encounters the outer boundary at $T_{2}$. As time proceeds from $T_{2}$ to $T_{1}$, the kinetic energy is gradually changed during the growth phase primarily by the drift betatron, but the gyro betatron becomes significant during the expansion phase. Because the drift betatron dominates the gyro betatron, the kinetic energy increases with time.

\section{Discussion}

\subsection{Substorm-Associated Electric Field}

[39] We have shown that a large-amplitude, highly fluctuating electric field is induced in the inner magnetosphere near the onset. Previously, a similar electric field was observed and interpreted in terms of the Rayleigh-Taylor instability 
EBIHARA AND TANAKA: IMPACT OF SUBSTORM ON ENERGETIC ELECTRON

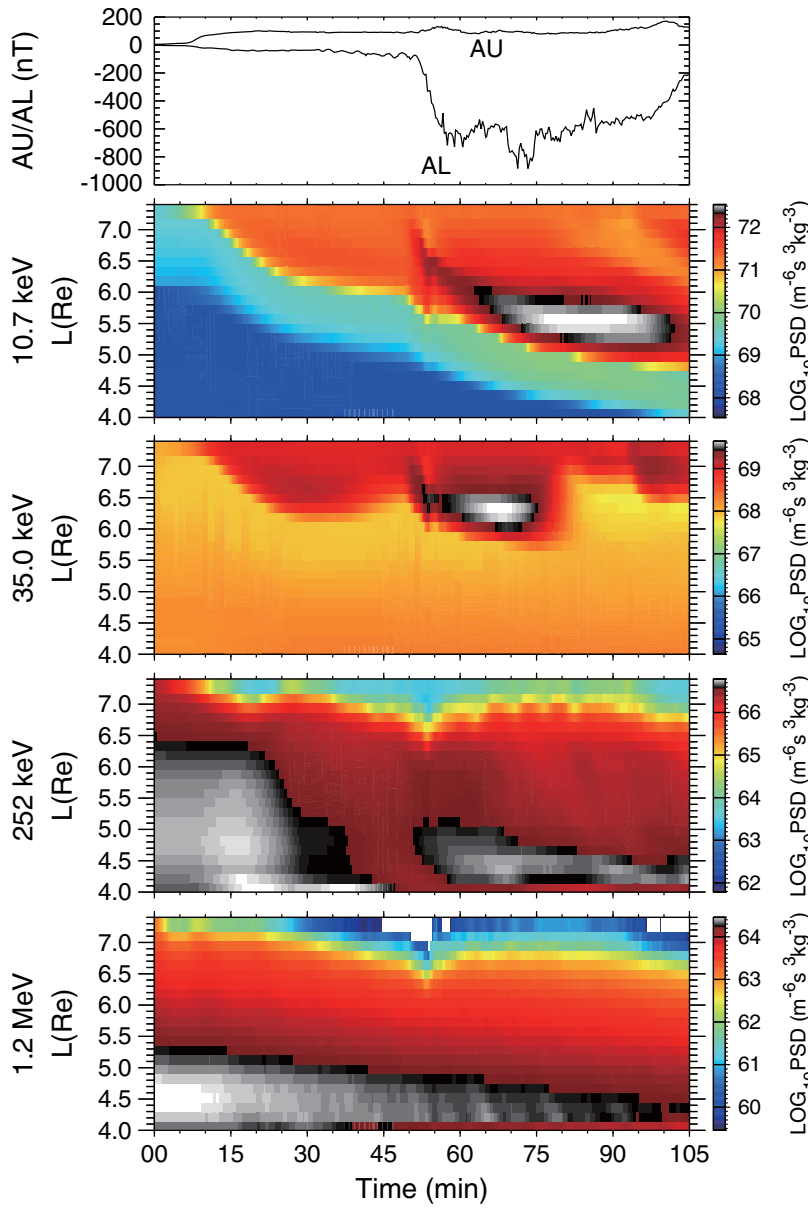

Figure 13. $L$-time diagrams of phase space density of electrons with energy of $10.7,35.0,252 \mathrm{keV}$, and $1.2 \mathrm{MeV}$ at midnight in the equatorial plane. The equatorial pitch angle of the electron is $58.2^{\circ}$. The calculated $A U / A L$ indices are shown at the top.

[Roux et al., 1991], a bouncing Alfvén wave [Maynard et al., 1996], and a fast magnetosonic mode wave propagating earthward [Sergeev et al., 1998]. Sergeev et al. [1998] have shown that the period of the oscillating electric field is approximately $1-2 \mathrm{~min}$, which is fairly consistent with our simulation. They also suggested that the oscillating electric field is a fast-magnetosonic-mode wave propagating earthward because the electric field variation associated with a substorm must be coherent at $\approx 5 \mathrm{Re}$ and $\approx 6.6 \mathrm{Re}$. The coherence of the oscillating electric field at $\approx 5 \mathrm{Re}$ and $\approx 6.6 \mathrm{Re}$ may be considered as the force imbalance that appears at $\approx 5 \mathrm{Re}$ and $\approx 6.6 \mathrm{Re}$, rather than the fast-mode wave, because their data show that $P$ and $B_{z}$ are anticorrelated with each other.

[40] Birn et al. [2011] found through global MHD simulations oscillating plasma flows in the near-Earth plasma sheet near the dipolarization. They interpreted the oscillation in terms of an expansion and thinning of the near-Earth magnetic field lines because of the upward (positive $Z$ ) and downward (negative $Z$ ) motion of the plasma. In contrast, our simulation shows that the oscillation in the $X$-direction dominates that in the $Z$-direction at $X \approx 6 \mathrm{Re}$. Thus, the

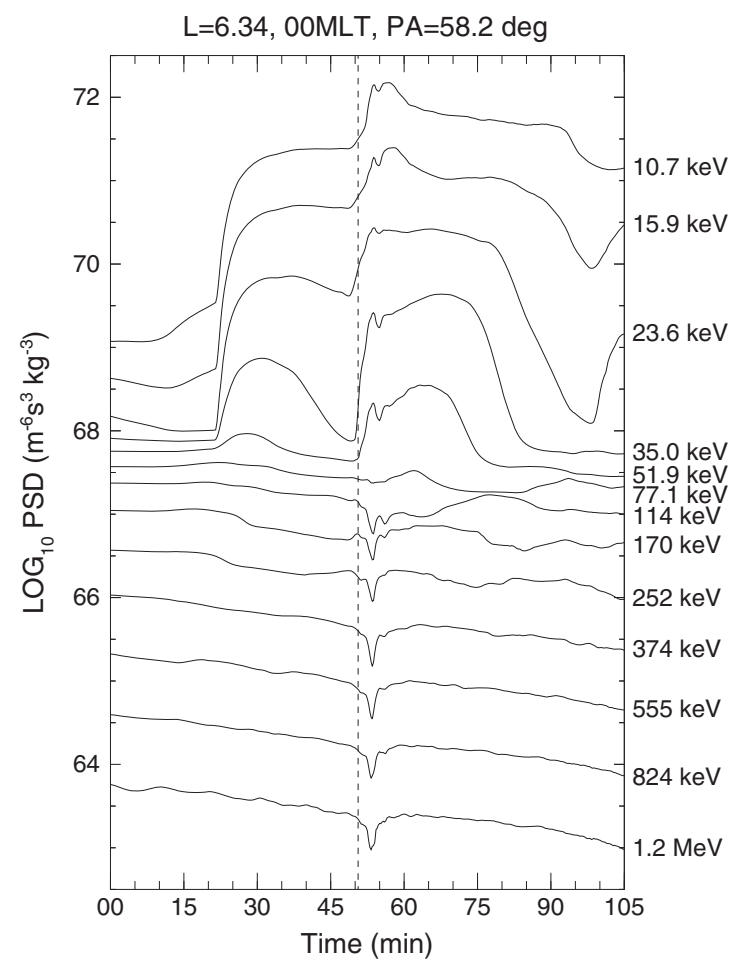

Figure 14. Phase space density of electrons with energy from $10.7 \mathrm{keV}$ to $1.2 \mathrm{MeV}$ at $L=6.34$ at midnight in the equatorial plane. The dashed line indicates the onset as determined by the calculated $A L$ index.

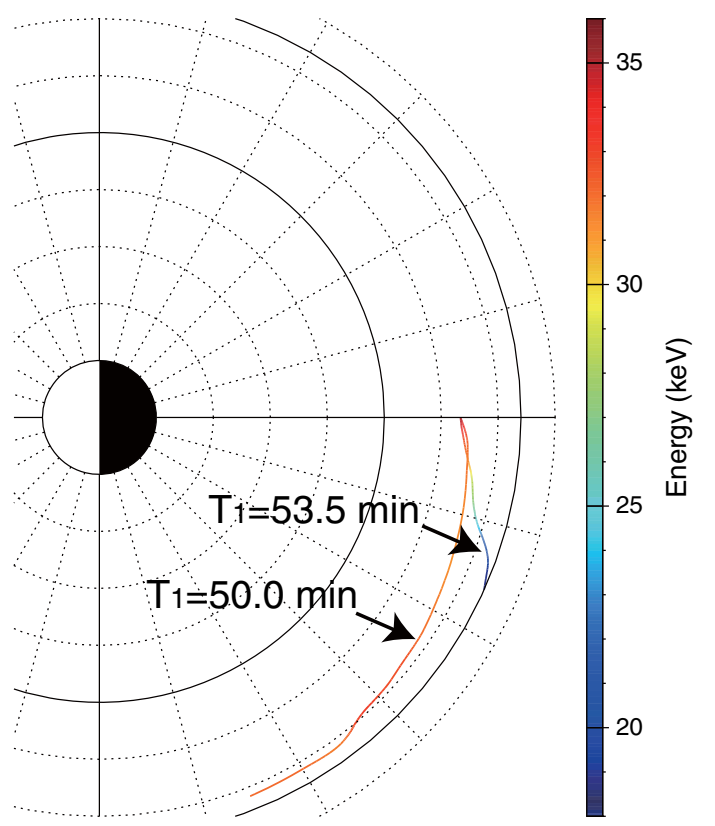

Figure 15. Bounce-averaged drift trajectories of the $35 \mathrm{keV}$ electron that arrives at point $S$ (at $6.34 \mathrm{Re}$ at midnight in the equatorial plane) at 50.0 and $53.5 \mathrm{~min}$. The kinetic energy of the electron is indicated by the color code.

large-amplitude, highly fluctuating electric field may be different in nature from the oscillating plasma flows reported by Birn et al. [2011]. 
EBIHARA AND TANAKA: IMPACT OF SUBSTORM ON ENERGETIC ELECTRON

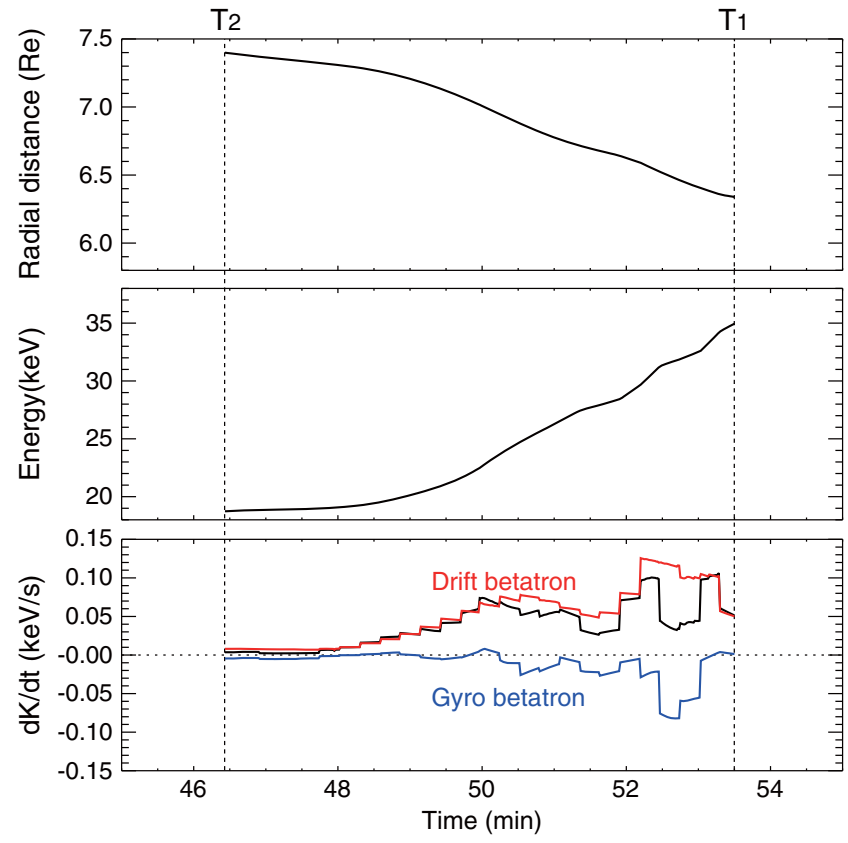

Figure 16. History of radial distance, kinetic energy, and energy gain rate of the $35 \mathrm{keV}$ electron that arrives at point $S$ at $53.5 \mathrm{~min}$. The red color indicates the energy gain rate due to the drift betatron, and the blue one indicates the energy gain rate due to the gyro betatron.

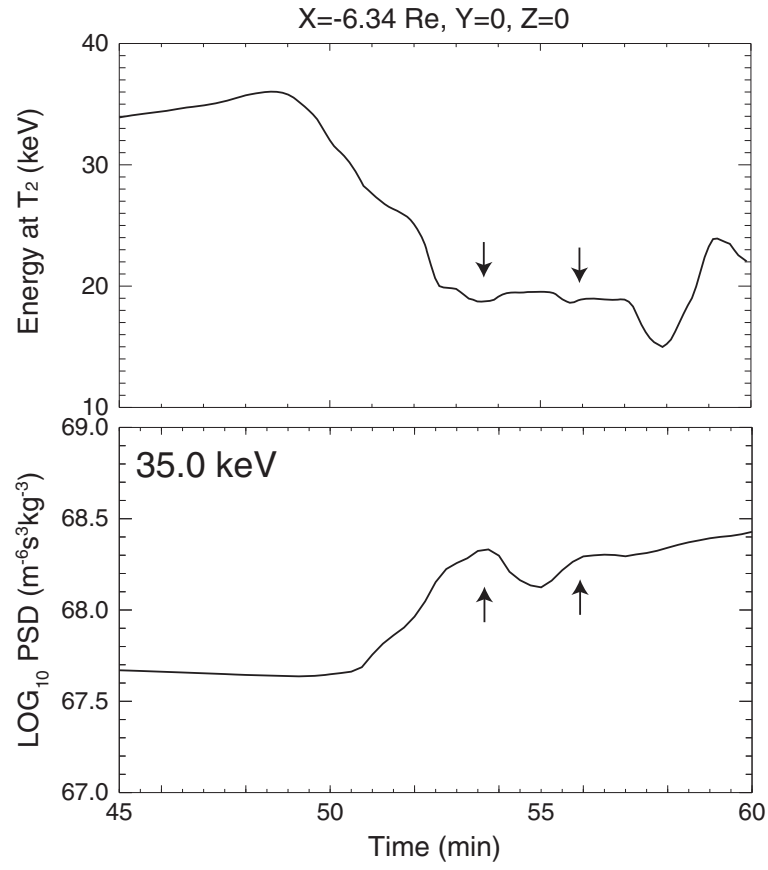

Figure 17. (top) Final kinetic energy of $35 \mathrm{keV}$ electron that arrives at point $S$ at $53.5 \mathrm{~min}$ (see the text) and (bottom) phase space density of the $35 \mathrm{keV}$ electron.

\subsection{Evolution of Low-Energy Electrons}

[41] The PSD of electrons with energy less than $52 \mathrm{keV}$ shows a two-step increase, and the second increase consists

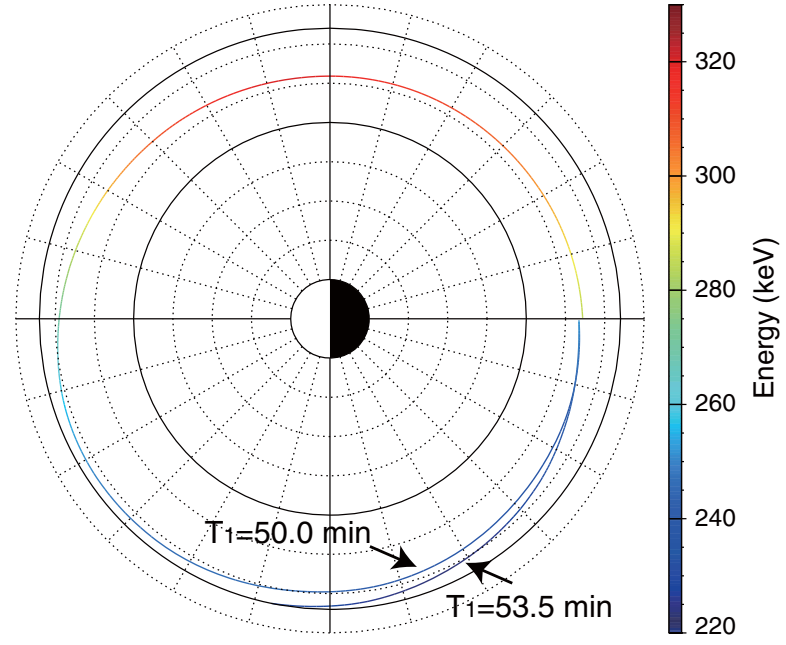

Figure 18. Same as Figure 15 except for $252 \mathrm{keV}$ electrons.
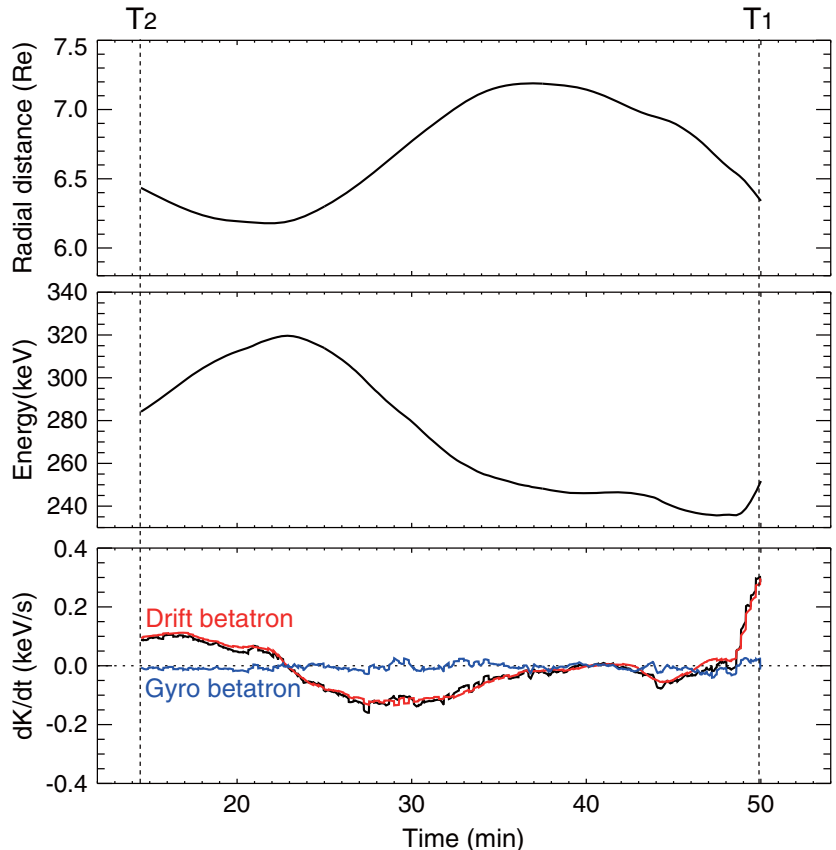

Figure 19. Same as Figure 16 except for the $252 \mathrm{keV}$ electron that arrives at point $S$ at $50 \mathrm{~min}$.

of a subset of multiple increases. The two-step increase results from the transportation of electrons from the outer boundary on the nightside. Thus, we can safely call them two-step injections. Multiple injections of energetic electrons have been observed at, or inside, the geosynchronous orbit [e.g., Baker et al., 1982b; Sergeev et al., 1998]. Sergeev et al. [1998] have speculated that the multiple injections are caused by the multiple impulsive $E_{y}$ associated with the fast-mode wave because the multiple injections occurred almost coherently at $L \approx 5$ and 6.6. Our simulation result shows that the impulsive injections are caused by two factors depending on location. One is directly caused by the force imbalance initiated by the sudden increase in the plasma pressure. The other is the fast-mode wave traveling from the region where the plasma pressure is suddenly increased. 


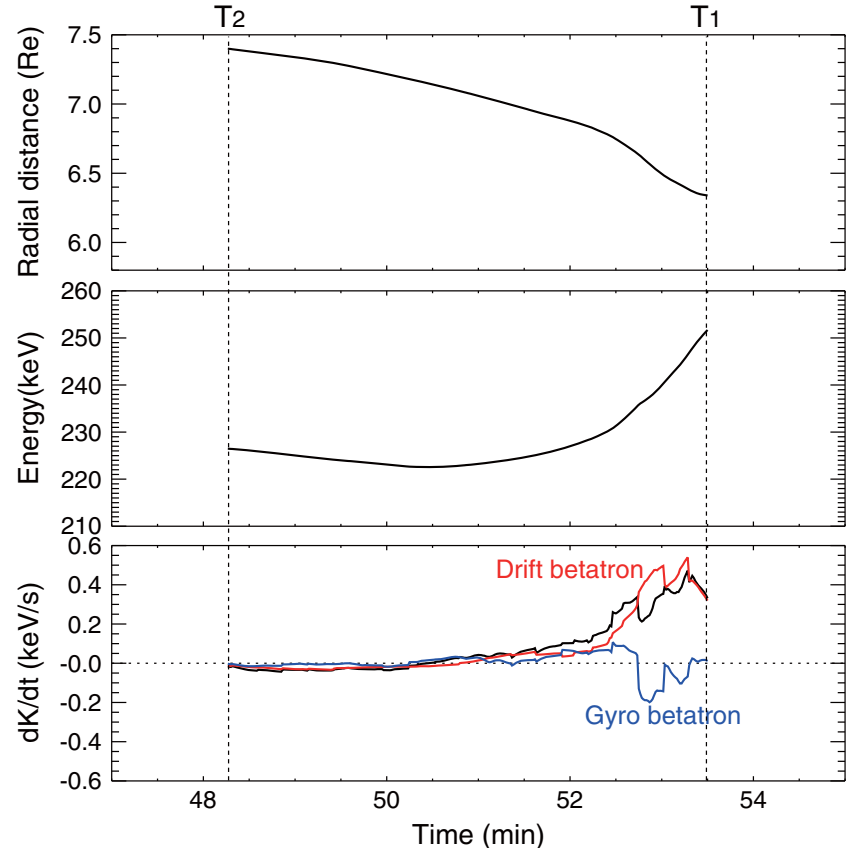

Figure 20. Same as Figure 16 except for the $252 \mathrm{keV}$ electron that arrives at point $S$ at $53.5 \mathrm{~min}$.

The former factor is associated with the negative correlation between $P$ and $B$, whereas the latter one is associated with the positive correlation between them. The data from CRRES show the negative correlation between them, which may favor the former factor.

\subsection{Evolution of High-Energy Electrons}

[42] The PSD of electrons with energy larger than $114 \mathrm{keV}$ shows a decrease during the growth phase of the substorm, which is consistent with observations near the geosynchronous orbit [e.g., Erickson and Winckler, 1973; Walker et al., 1976; Erickson et al., 1979; Sauvaud and Winckler, 1980; Nagai, 1982; Birn et al., 1997]. Our simulation result shows that the decrease in the $252 \mathrm{keV}$ electron is primarily caused by the adiabatic deceleration due to the drift betatron under the presence of the eastward induction electric field as also pointed out by Sauvaud et al. [1996]. The radial motion of the electrons is not significant. This conclusion can be applied to the electrons with energy higher than $252 \mathrm{keV}$. As for electrons with energy ranging between 114 and $252 \mathrm{keV}$, the adiabatic deceleration due to the gyro betatron becomes effective in the late growth phase, but the drift betatron still dominates the gyro betatron. The PSD of the electrons also shows a decrease (a dip) during the expansion phase, which is caused by the boundary shadowing. That is, the electron encounters the outer boundary as it drifts eastward and cannot complete the circular motion around the Earth. Nagai [1982] found that the region of the decrease in the $>2 \mathrm{MeV}$ electron flux during the expansion phase is restricted to the afternoon side. Our current simulation cannot explain the decrease in the electron flux on the afternoon side during the expansion phase.

[43] Sometimes, energetic electrons of a few hundred $\mathrm{keV}$ significantly increase immediately after the onset of substorms and exceed the pre-substorm level [e.g., Erickson et al., 1979; Baker et al., 1982a]. Such enhancements can be understood in terms of the betatron acceleration that occurs at the dipolarization front propagating earthward in the nearEarth plasma sheet, according to test particle simulations in the electric and magnetic fields given by the global MHD simulation [Birn et al., 1998; Ashour-Abdalla et al., 2011]. This process cannot be handled by our simulation because the bounce-averaged approximation is invalid. In the near future, we will develop a test particle simulation to address the acceleration processes that occur in the dipolarization front.

[44] Nagai et al. [2006] reported a substantial enhancement of the relativistic electron flux $(>2.5 \mathrm{MeV})$ at $L \sim 3$ after a storm-time substorm on a timescale of less than 60 min. The coupled Radiation Belt Environment and Block-Adaptive-Tree Solar Wind Roe-Type Upwind Scheme simulation shows that the relativistic electron flux exceeds that given by the AE8 model owing to the induction electric field associated with a substorm [Glocer et al., 2011]. Our drift kinetic simulation cannot show such an enhancement of the $\mathrm{MeV}$ electrons. One possibility is that the electric field simulated by the current MHD simulation is too weak to influence the $\mathrm{MeV}$ electrons.

\section{Conclusions}

[45] 1. Two types of the electric field are generated in the inner magnetosphere at $<7 \mathrm{Re}$ during a substorm. The first one is generated several to ten minutes after the southward turning of IMF. The other one is generated near the onset, which oscillates with a period of approximately $2-3 \mathrm{~min}$. The latter electric field results from the imbalance between the $J \times B$ force and the $\nabla P$ force in the vicinity of the pressure peak at $L \approx 6-7$. The pressure-driven electric field may propagate toward the Earth in the inner magnetosphere as a fast-mode magnetosonic wave.

[46] 2. The PSD of electrons at energies equal to, or less than, $51.9 \mathrm{keV}$ shows a two-step injection. The first injection is initiated several to ten minutes after the southward turning. The second injection occurs near the onset and consists of a subset of multiple injections, which is caused by the oscillating electric field.

[47] 3. The PSD of electrons at energies equal to, or greater than, $114 \mathrm{keV}$ shows a two-step decrease. The first decrease starts just after the southward turning of IMF, which results from deceleration of the electron under the influence of the eastward induction electric field, not a radial displacement. The second decrease occurs near the onset, which is caused by boundary shadowing.

[48] 4. All these variations of the PSD of electrons are understood to be a natural consequence of the state transition of the magnetosphere-ionosphere system that responds to the solar wind condition.

\section{Appendix A: Derivation of Equation (1)}

[49] Following Jordanova et al. [2006], we start by describing the perpendicular and parallel components of the momentum at a given position along a field line as

$$
\begin{aligned}
p_{\perp}(s) & =p \sin \alpha \\
& =p \sqrt{\frac{B(s)}{B_{0}}\left(1-x^{2}\right),}
\end{aligned}
$$


EBIHARA AND TANAKA: IMPACT OF SUBSTORM ON ENERGETIC ELECTRON

$$
\begin{aligned}
p_{\|}(s) & =p \cos \alpha \\
& =p \sqrt{1-\frac{B(s)}{B_{0}}\left(1-x^{2}\right)},
\end{aligned}
$$

respectively, where $p=$ momentum, $\alpha=$ pitch angle, $B=$ magnetic field, $B_{0}=$ magnetic field in the equatorial plane, and $x=$ cosine of the equatorial pitch angle. The Jacobian transformation of equations (A1) and (A2) is given by

$$
\frac{\partial\left(p_{\perp}, p_{\|}\right)}{\partial(K, x)}=\frac{\frac{B(s)}{B_{0}} m \gamma p x}{p_{\perp} \sqrt{1-\frac{B(s)}{B_{m}}}}
$$

where $K=$ kinetic energy, $m=$ mass, $\gamma=$ Lorentz factor, and $B_{m}=$ magnetic field at the mirror point.

[50] A volume element in phase space is, by definition, given by

$$
d V=d^{3} \mathbf{r} d^{3} \mathbf{p}
$$
by

[51] The volume element in configuration space is given

$$
d^{3} \mathbf{r}=\frac{B_{0}}{B(s)} R d R d \varphi d s
$$

where $R$ is the radial distance, $\varphi$ is the local time in the equatorial plane and $s$ is a line element along a field line. Ignoring the gyrophase, we can obtain the volume element in momentum space:

$$
d^{3} \mathbf{p}=4 \pi p_{\perp} d p_{\perp} d p_{\|}
$$

[52] The volume element in momentum space is doubled because of its symmetry with respect to a pitch angle of $\pi / 2$. Considering (A3), the volume element can be rewritten as

$$
d V=4 \pi m S_{b}(R, \varphi, x) R \gamma p x d R d \varphi d K d x,
$$

where $S_{b}$ is a function given by (2).

[53] Assuming conservation of the number of particles, one can write the drift kinetic equation of the four-dimensional phase space density $f(R, \varphi, K, x)$ as

$$
\begin{aligned}
\frac{\partial f}{\partial t} & +\frac{1}{R S_{b}} \frac{\partial}{\partial R}\left(R S_{b}\left\langle\frac{d R}{d t}\right\rangle f\right)+\frac{1}{S_{b}} \frac{\partial}{\partial \varphi}\left(S_{b}\left\langle\frac{d \varphi}{d t}\right\rangle f\right) \\
& +\frac{1}{\gamma p} \frac{\partial}{\partial K}\left(\gamma p\left\langle\frac{d K}{d t}\right\rangle f\right)+\frac{1}{x S_{b}} \frac{\partial}{\partial x}\left(x S_{b} \frac{d x}{d t} f\right)=\left(-\frac{2 f}{\tau_{b}}\right)_{\text {loss cone }},
\end{aligned}
$$

where $\tau_{b}$ is the bounce period. The right-hand side of equation (A8) implies that particles are lost on a timescale half that of the bounce period in the loss cone. Equations (A7) and (A8) are different from those used by Jordanova et al. [2006], who assumed that the value of $h$ (which is equal to the value of $S_{b} / R$ ) is independent of $R$ and $\varphi$. Obviously, $h$ depends on $R$ and $\varphi$ in a realistic magnetic field.

\section{Appendix B: Derivation of Equation (9)}

[54] Differentiating both sides of equation (7) with respect to time, we obtain

$$
\begin{aligned}
\frac{d x}{d t} & =\frac{1-x^{2}}{x p} \frac{d p}{d t}-\frac{m M}{x p^{2}} \frac{d B}{d t} \\
& =\frac{1-x^{2}}{x}\left(\frac{1}{p} \frac{d p}{d t}-\frac{1}{2 B} \frac{d B}{d t}\right),
\end{aligned}
$$

where $m=$ mass, $M=$ magnetic moment of the particle, $B=$ magnetic field in the equatorial plane, $p=$ momentum, and $x=$ cosine of the equatorial pitch angle. Considering the equation

$$
\frac{d B}{d t}=\frac{\partial B}{\partial t}+\langle\mathbf{V}\rangle \bullet \nabla B
$$

we rewrite equation $(\mathrm{B} 1)$ as

$$
\begin{aligned}
\frac{d x}{d t} & =\frac{1-x^{2}}{x}\left[\frac{1}{p} \frac{d p}{d t}-\frac{1}{2 B}\left(\frac{\partial B}{\partial t}+\langle\mathbf{V}\rangle \bullet \nabla B\right)\right] \\
& =\frac{1-x^{2}}{x}\left[\frac{K+m c^{2}}{K^{2}+2 m c^{2} K} \frac{d K}{d t}-\frac{1}{2 B}\left(\frac{\partial B}{\partial t}+\langle\mathbf{V}\rangle \bullet \nabla B\right)\right] .
\end{aligned}
$$

[55] Acknowledgments. We thank Takashi Kikuchi, Tsugunobu Nagai, Shigeru Fujita, and Kunihiko Keika for the fruitful comments and discussion. This study was supported by KAKENHI, a Grant-in-Aid for Young Scientists (B) 21740354 and a Grant-in-Aid for Scientific Research (B) 24340119

\section{References}

Arnoldy, R. L., and K. W. Chan (1969), Particle substorms observed at the geostationary orbit, J. Geophys. Res., 74(21), 5019-5028, doi:10.1029/ JA074i021p05019.

Ashour-Abdalla, M., et al. (2011), Observations and simulations of nonlocal acceleration of electrons in magnetotail magnetic reconnection events, Nat. Phys., 7, 360-365, doi:10.1038/nphys 1903.

Baker, D. N., et al. (1982a), Observation and modeling of energetic particles at synchronous orbit on July 29, 1977, J. Geophys. Res., 87(A8), 5917-5932.

Baker, D. N., E. W. Hones Jr., R. D. Belian, P. R. Higbie, R. P. Lepping, and P. Stauning (1982b), Multiple-spacecraft and correlated riometer study of magnetospheric substorm phenomena, J. Geophys. Res., 87(A8), 6121-6136, doi:10.1029/JA087iA08p06121.

Birn, J., M. F. Thomsen, J. E. Borovsky, G. D. Reeves, D. J. McComas, and R. D. Belian (1997), Characteristic plasma properties during dispersionless substorm injections at geosynchronous orbit, J. Geophys. Res., 102(A2), 2309-2324

Birn, J., M. F. Thomsen, J. E. Borovsky, G. D. Reeves, D. J. McComas, R. D. Belian, and M. Hesse (1998), Substorm electron injections: geosynchronous observations and test particle simulations, J. Geophys. Res. 103(A5), 9235-9248, doi:10.1029/97JA02635.

Birn, J., R. Nakamura, E. V. Panov, and M. Hesse (2011), Bursty bulk flows and dipolarization in MHD simulations of magnetotail reconnection, J. Geophys. Res., 116, A01210, doi:10.1029/2010JA016083.

Christon, S., D. Williams, D. Mitchell, C. Huang, and L. Frank (1991), Spectral characteristics of plasma sheet ion and electron populations during disturbed geomagnetic conditions, J. Geophys. Res., 96(A1), 1-22.

Davis, T. N., and M. Sugiura (1966), Auroral electrojet activity index AE and its universal time variations, J. Geophys. Res., 71, 785-801.

DeForest, S. E., and C. E. McIlwain (1971), Plasma clouds in the magnetosphere, J. Geophys. Res., 76(16), 3587-3611, doi:10.1029/JA076i016p03587. De Zeeuw, D. L., T. I. Gombosi, C. P. T. Groth, K. G. Powell, and Q. F. Stout (2000), An adaptive MHD method for global space weather simulations, IEEE Trans. Plasma Sci., 28, 1956-1965, doi:10.1109/27.902224.

Erickson, K. N., and J. R. Winckler (1973), Auroral electrojets and evening sector electron dropouts at synchronous orbit, J. Geophys. Res., 78(34), 8373-8380, doi:10.1029/JA078i034p08373. 


\section{EBIHARA AND TANAKA: IMPACT OF SUBSTORM ON ENERGETIC ELECTRON}

Erickson, K. N., R. L. Swanson, R. J. Walker, and J. R. Winckler (1979), A study of magnetosphere dynamics during auroral electrojet events by observations of energetic electron intensity changes at synchronous orbit, J. Geophys. Res., 84(A3), 931-942, doi:10.1029/JA084iA03p00931.

Fok, M.-C., T. E. Moore, and D. C. Delcourt (1999), Modeling of inner plasma sheet and ring current during substorms, J. Geophys. Res., 104(A7), 14557-14569, doi:10.1029/1999JA900014.

Fok, M.-C., et al. (2001), Comprehensive computational model of earth's ring current, J. Geophys. Res., 106(A5), 8417-8424.

Fok, M.-C., et al. (2008), Radiation Belt Environment model: application to space weather nowcasting, J. Geophys. Res., 113, A03S08, doi:10.1029/2007JA012558

Freeman, J. W., Jr., and J. J. Maguire (1967), Gross local-time particle asymmetries at the synchronous orbit altitude, J. Geophys. Res., 72(21), 5257-5264, doi:10.1029/JZ072i021p05257.

Friedel, R. H. W., A. Korth, and G. Kremser (1996), Substorm onsets observed by CRRES: determination of energetic particle source regions, J. Geophys. Res., 101(A6), 13,137-13,154, doi:10.1029/96JA00399.

Glocer, G., M.-C. Fok, T. Nagai, G. Tóth, T. Guild, and J. Blake (2011), Rapid rebuilding of the outer radiation belt, J. Geophys. Res., 116, A09213, doi:10.1029/2011JA016516.

Gombosi, T. I., G. Tóth, D. L. de Zeeuw, K. G. Powell, and Q. F. Stout (2003), Adaptive mesh refinement MHD for global simulations, in Space Plasma Simulation, Lect. Notes Phys., vol. 615, edited by J. Büchner, C. T. Dum, and M. Scholer, pp. 251-279, Springer, Berlin.

Hardy, D. A., M. S. Gussenhoven, and E. Holeman (1985), A statistical model of auroral electron precipitation, J. Geophys. Res., 90(A5), 4229-4248, doi:10.1029/JA090iA05p04229.

Hones, E. W., Jr., S. Singer, and C. S. R. Rao (1968), Simultaneous observations of electrons $(\mathrm{E}>45 \mathrm{keV})$ at 2000-kilometer altitude and at 100,000 kilometers in the magnetotail, J. Geophys. Res., 73(23), 7339-7359, doi:10.1029/JA073i023p07339.

Jordanova, V. K., Y. S. Miyoshi, S. Zaharia, M. F. Thomsen, G. D. Reeves, D. S. Evans, C. G. Mouikis, and J. F. Fennell (2006), Kinetic simulations of ring current evolution during the Geospace Environment Modeling challenge events, J. Geophys. Res., 111, A11S10, doi:10.1029/2006JA011644.

Kim, H. J., A. A. Chan, R. A. Wolf, and J. Birn (2000), Can substorms produce relativistic outer-belt electrons?, J. Geophys. Res., 105, 7721-7736.

Lax, P. D., and B. Wendroff (1960), Systems of conservation laws, Commun. Pure Appl. Math., 13, 217-237.

Li, X., D. N. Baker, M. Temerin, G. Reeves, and R. Belian (1998), Simulation of dispersionless injections and drift echoes of energetic electrons associated with substorms, Geophys. Res. Lett., 25, 3736-3766.

Lopez, R. E., D. G. Sibeck, R. W. McEntire, and S. M. Krimigis (1990), The energetic ion substorm injection boundary, J. Geophys. Res., 95(A1), 109-117, doi:10.1029/JA095iA01p00109.

Lui, A. T. Y., K. Liou, M. Nosé, S. Ohtani, D. J. Williams, T. Mukai, K. Tsuruda, and S. Kokubun (1999), Near-Earth dipolarization: evidence for a non-MHD process, Geophys. Res. Lett., 26(19), 2905-2908, doi:10.1029/1999GL003620.

Maynard, N. C., W. J. Burke, E. M. Basinska, G. M. Erickson, W. J. Hughes, H. J. Singer, A. G. Yahnin, D. A. Hardy, and F. S. Mozer (1996), Dynamics of the inner magnetosphere near times of substorm onsets, J. Geophys. Res. 101(A4), 7705-7736, doi:10.1029/95JA03856.

McIlwain, C. E. (1974), Substorm injection boundaries, in Magnetospheric Physics, edited by B. M. McCormac, p. 143, D. Reidel, Norwell, Mass.

Nagai, T. (1982), Local time dependence of electron flux changes during substorms derived from multi-satellite observation at synchronous orbit, J. Geophys. Res., 87(A5), 3456-3468, doi:10.1029/JA087iA05p03456.

Nagai, T. (1988), "Space weather forecast": prediction of relativistic electron intensity at synchronous orbit, Geophys. Res. Lett., 15, 425-428.

Nagai, T., A. S. Yukimatu, A. Matsuoka, K. T. Asai, J. C. Green, T. G. Onsager, and H. J. Singer (2006), Timescales of relativistic electron enhancements in the slot region, J. Geophys. Res., 111, A11205, doi:10.1029/2006JA011837.
Powell, K., P. Roe, T. Linde, T. Gombosi, and D. L. De Zeeuw (1999), A solution-adaptive upwind scheme for ideal magnetohydrodynamics, J. Comput. Phys., 154, 284-309, doi:10.1006/jcph.1999.6299.

Reeves, G. D., M. G. Henderson, P. S. McLachlan, R. D. Belian, R. H. W. Friedel, and A. Korth (1996), Radial propagation of substorm injections, in Proceedings of the Third International Conference on Substorms, Versailles, France, 12-17 May 1996, Eur. Space Agency Spec. Publ., ESA SP-389, 579-584.

Roe, P. L. (1985), Some contributions to the modelling of discontinuous flows, in B.E. Engquist, S. Osher, and R.C.J. Somerville, editors, LargeScale Computations in Fluid Mechanics, volume 22 of Lectures in Applied Mathematics, pp. 163-193. American Mathematical Society, Providence, RI.

Roederer, J. G. (1970), Dynamics of Geomagnetically Trapped Radiation, pp. 49-74, Springer-Verlag, Berlin

Rostoker, G., S.-I. Akasofu, J. Foster, R. A. Greenwald, Y. Kamide K. Kawasaki, A. T. Y. Lui, R. L. McPherron, and C. T. Russell (1980), Magnetospheric substorms-definition and signatures, J. Geophys. Res., 85(A4), 1663-1668.

Roux, A., S. Perraut, P. Robert, A. Morane, A. Pedersen, A. Korth, G. Kremser, B. Aparicio, D. Rodgers, and R. Pellinen (1991), Plasma sheet instability related to the westward traveling surge, J. Geophys. Res., 96(A10), 17,697-17,714, doi:10.1029/91JA01106.

Sauvaud, J.-A., and J.R. Winckler (1980), Dynamics of plasma, energetic particles, and fields near synchronous orbit in the nighttime sector during magnetospheric substorms, J. Geophys. Res., 85(A5), 2043-2056, doi:10.1029/JA085iA05p02043.

Sauvaud, J. A., T. Beutier, and D. Delcourt (1996), On the origin of flux dropouts near geosynchronous orbit during the growth phase of substorms: 1. Betatron effects, J. Geophys. Res., 101(A9), 19,911-19,919, doi:10.1029/96JA01632

Sergeev, V., M. Shukhtina, R. Rasinkangas, A. Korth, G. Reeves, H. Singer, M. Thomsen, and L. Vagina (1998), Event study of deep energetic particle injections during substorm, J. Geophys. Res., 103(A5), 9217-9234.

Tanaka, T. (1995), Generation mechanisms for magnetosphere-ionosphere current systems deduced from a three-dimensional MHD simulation of the solar wind-magnetosphere-ionosphere coupling processes, J. Geophys. Res., 100(A7), 12057-12074, doi:10.1029/95JA00419.

Tanaka, T., A. Nakamizo, A. Yoshikawa, S. Fujita, H. Shinagawa, H. Shimazu, T. Kikuchi, and K. K. Hashimoto (2010), Substorm convection and current system deduced from the global simulation, J. Geophys. Res., 115, A05220, doi:10.1029/2009JA014676.

Vasyliunas, V. M. (1968), A survey of low-energy electrons in the evening sector of the magnetosphere with OGO 1 and OGO 3, J. Geophys. Res., 73,2839

Vasyliunas, V. M. (1970), Mathematical models of magnetospheric convection and its coupling to the ionosphere, in Particles and Fields in the Magnetosphere, edited by B. McCormac, p. 60, D. Reidel, Norwell, Mass.

Vette, J. I. (1991), The AE-8 trapped electron model environment. In: NSSDC WDC-A-R\&S 91-24, NASA-GSFC.

Walker, R. J., K. N. Erickson, R. L. Swanson, and J. R. Winckler (1976), Substorm-associated particle boundary motion at synchronous orbit J. Geophys. Res., 81(31), 5541-5550, doi:10.1029/JA081i031p05541.

Wolf, R. A. (1970), Effects of ionospheric conductivity on convection flow of plasma in the magnetosphere, J. Geophys. Res., 75, 4677.

Xiao, F., R. M. Thorne, and D. Summers (1998), Instability of electromagnetic r-mode waves in a relativistic plasma, Phys. Plasmas, 5, 2489.

Zhang, J.-C., R. A. Wolf, R. W. Spiro, G. M. Erickson, S. Sazykin, F. R. Toffoletto, and J. Yang (2009), Rice Convection Model simulation of the substorm-associated injection of an observed plasma bubble into the inner magnetosphere: 2. Simulation results, J. Geophys. Res., 114, A08219, doi:10.1029/2009JA014131.

Zheng, Y., M.-C. Fok, and G. V. Khazanov (2003), A radiation belt-ring current forecasting model, Space Weather, 1(3), 1013, doi:10.1029/ 2003SW000007. 